\title{
Asymmetric electrochemical oxidation of 1,2-diols, aminoalcohols and aminoaldehydes in the presence of chiral copper catalyst
}

\author{
Daishirou Minato, Hitomi Arimoto, Yoko Nagasue, Yosuke Demizu and Osamu \\ Onomura* $^{*}$ \\ Graduate School of Biomedical Sciences, Nagasaki University, \\ 1-14 Bunkyo-machi, Nagasaki 852-8521, Japan
}

\begin{abstract}
Asymmetric oxidation of 1,2-diols, aminoalcohols and aminoaldehydes in the presence of copper(II) triflate and $(R, R)-\mathrm{Ph}-\mathrm{BOX}$, was accomplished by electrochemical method using $\mathrm{Br}^{-}$as a mediator. This oxidation was applicable to kinetic resolution of racemic cis-cycloalkane-1,2-diols, aminoalcohols and aminoaldehydes to afford optically active compounds with good to high enantioselectivity.
\end{abstract}

Key words: Asymmetric electrochemical oxidation; Copper complex; 1,2-Diol; $\alpha$-Ketoalcohol; Aminoalcohol; Aminoaldehyde

\section{Introduction}

Selective oxidation of hydroxyl group to carbonyl group is a basic and important organic reaction. It was reported in 1974 that 1,2-diols are selectively oxidized to the corresponding $\alpha$-ketoalcohols by utilizing a stoichiometric amount of dibutyltinoxide $\left(\mathrm{Bu}_{2} \mathrm{SnO}\right)$ which forms dibutylstannylenes followed by brominolysis. ${ }^{1}$ From the standpoint of green chemistry, we have reported efficient oxidation of 1,2-diols A by electrochemical method using a catalytic amount of $\mathrm{Bu}_{2} \mathrm{SnO}$ and $\mathrm{Br}^{-}$ion to afford $\alpha$-ketoalcohols B in high yield without 1,2-diketones C (Eq. 1). ${ }^{2}$

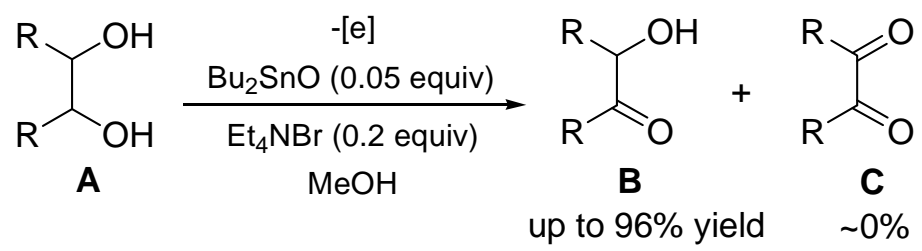

\footnotetext{
${ }^{*}$ Corresponding author, Tel +81-95-819-2429, Fax +81-95-819-2476, E-mail: onomura@nagasaki-u.ac.jp
} 
More recently, we reported the first catalytic asymmetric oxidation of 1,2-diols A' to afford the corresponding optically active $\alpha$-ketoalcohols B' in high enantioselectivity, ${ }^{3}$ which is based on recognition of the diol-moiety by copper(II) ion associated with $(R, R)-\mathrm{Ph}-\mathrm{BOX}$ complex ${ }^{4}$ to form the activated intermediates $\mathbf{D}$ ' followed by oxidation with NBS (Eq. 2). So far, to the best of our knowledge, catalytic asymmetric oxidation of $\mathbf{A}^{\prime}$ to $\mathbf{B}^{\prime}$ has not been known except for two examples using semi-catalytic amount of chiral dioxiranes ${ }^{5}$ or chiral hypervalent iodine. ${ }^{6}$

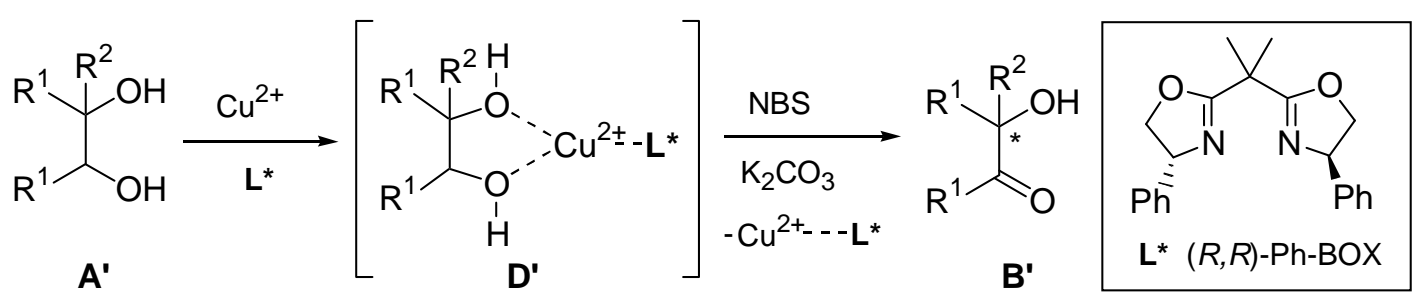

In continuing the study, we succeeded in kinetic resolution of $\mathbf{A}^{\prime}$ by electrochemical oxidation. The concept we used in the asymmetric electrochemical oxidation of $\mathbf{A}^{\mathbf{\prime}}$ to optically active $\alpha$-ketoalcohols $\mathbf{B}^{\prime}$ is schematically represented in Scheme 1. Complex D' consisting diols A' and chiral copper catalyst $\mathrm{Cu}-\mathbf{L}^{*}$ was easily deprotonated by cathodically generated $\mathrm{MeO}^{-}$to afford alkoxide anions $\mathbf{E}^{\prime}$, which reacted with anodically generated $\mathrm{Br}^{+}$to form $\mathrm{O}$-brominated intermediates $\mathbf{F}^{\prime}$. $\mathrm{MeO}^{-}$removed $\mathrm{HBr}$ from $\mathbf{F}^{\prime}$ to afford $\mathbf{B}^{\prime}$ and regenerate $\mathrm{Cu}-\mathbf{L}^{*}$. Although a mechanism for this oxidation is similar to that of the oxidation with $\mathrm{NBS}^{3}{ }^{3}$ this electrochemical method is effective for some diols that NBS could not oxidize. In addition, this method is applicable to the asymmetric oxidation of aminoalcohols $\mathbf{G}^{\prime}$ and aminoaldehydes $\mathbf{H}^{\prime}$ to the corresponding optically active aminoesters. 


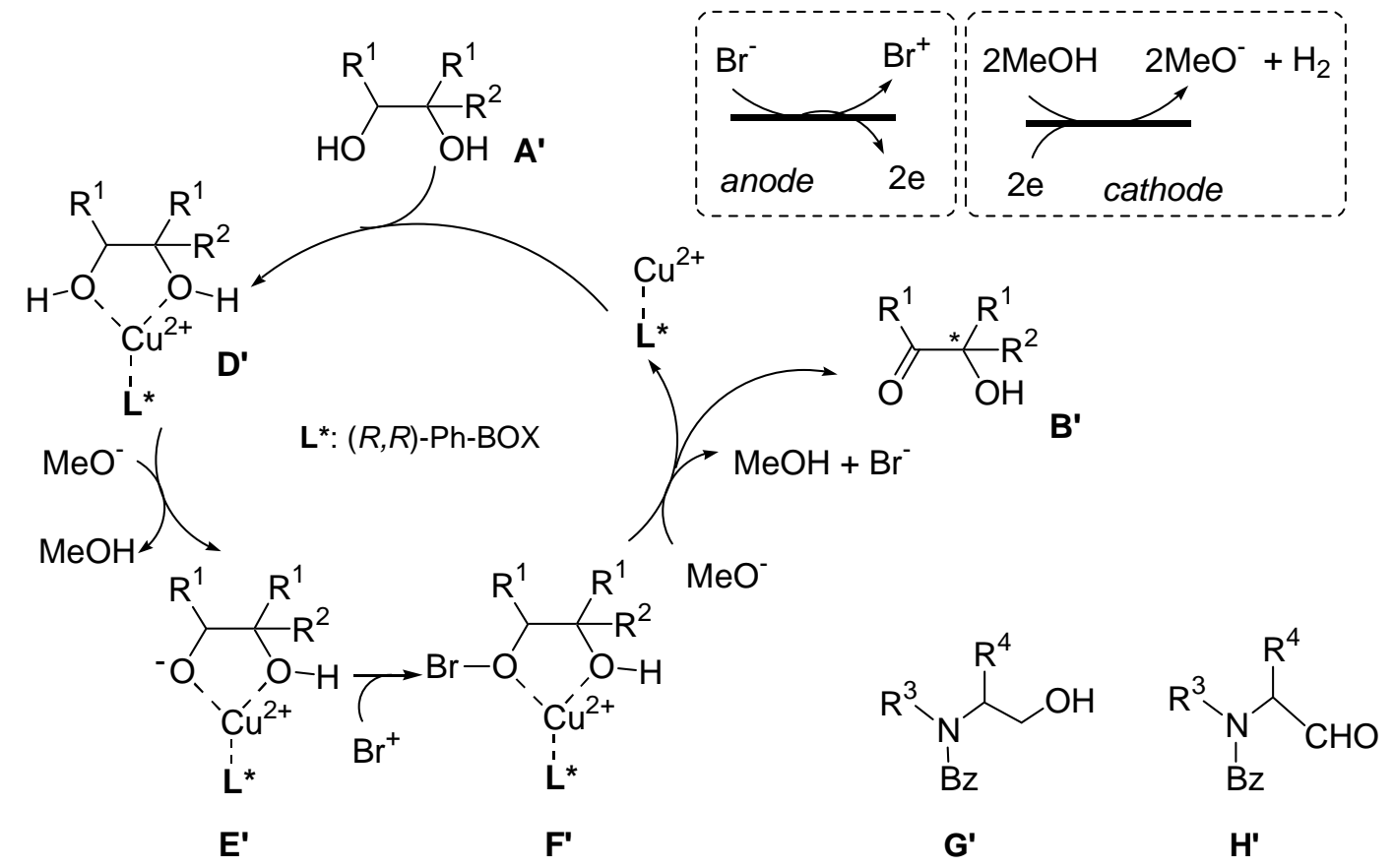

Scheme 1

\section{Results and discussion}

2.1 Accelerating effect based on recognition of the diol-moiety under the electrochemical oxidation condition

First, we tried electrochemical oxidation of phenylcyclohexane-cis-1,2-diol (cis-1) as a model compound to check for accelerating effect based on recognition of cis-1 with the $\mathrm{Cu}(\mathrm{II})-(R, R)-\mathrm{Ph}-\mathrm{BOX}$ complex. The electrochemical oxidation of cis-1 in the presence of $\mathrm{Cu}(\mathrm{OTf})_{2}$ and $(R, R)-\mathrm{Ph}-\mathrm{BOX}$ predominantly afforded mono-oxidized product 2 (36\% yield). However, the oxidation did not proceed in the absence of $\mathrm{Cu}(\mathrm{OTf})_{2}$ and $(R, R)-\mathrm{Ph}-\mathrm{BOX}$ (Eq. 3). These results suggests that cis-1 might be recognized by the $\mathrm{Cu}(\mathrm{II})-(R, R)-\mathrm{Ph}-\mathrm{BOX}$ complex under this oxidation condition. 


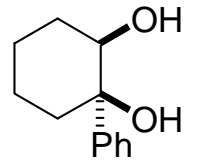

1

in the presence of $\mathrm{Cu}(\mathrm{OTf})_{2}$ (0.1 equiv) and $(R, R)-\mathrm{Ph}-\mathrm{BOX}$ (0.1 equiv)

in the presence of $\mathrm{Cu}(\mathrm{OTf})_{2}$ (0.1 equiv)

in the absence of $\mathrm{Cu}(\mathrm{OTf})_{2}$ and $(R, R)-\mathrm{Ph}-\mathrm{BOX}$

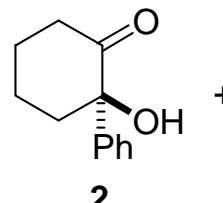

2

$36 \%$

$3 \%$

$0 \%$
$\overbrace{\overline{\mathrm{P} h}}^{\mathrm{OH}}$

recovered 1

$58 \%$

$88 \%$

$100 \%$

\subsection{Effects of electrolytes and solvents on asymmetric oxidation}

Next, we investigated electrolytes and solvents for the electrochemical oxidation applicable to kinetic resolution of cis-1 (Eq. 4). The results are summarized in Table 1. A procedure for asymmetric electrochemical oxidation of cis-1 is as follows; Anodic oxidation of 1 was carried out using platinum electrodes $\left(2 \times 1 \mathrm{~cm}^{2}\right)$ in an undivided beaker-type cell containing $0.5 \mathrm{mmol}$ of $\mathbf{1}, 1.0$ equiv of electrolyte, 0.1 equiv of $\mathrm{Cu}(\mathrm{OTf})_{2}, 0.1$ equiv of $(R, R)-\mathrm{Ph}-\mathrm{BOX}$, and solvent $(5 \mathrm{~mL})$. After passing through 2.0 $\mathrm{F} / \mathrm{mol}$ of electricity at constant current $(50 \mathrm{~mA})$ at $0^{\circ} \mathrm{C}$ and usual workup, optically active $\alpha$-ketoalcohol (S)-2 was obtained in $36 \%$ yield with moderate selectivity (s) value of 8 (Table 1 , entry 1$){ }^{6-9}$ Comparable result was obtained by use of $\mathrm{CuBr}_{2}$ instead of $\mathrm{Cu}(\mathrm{OTf})_{2}$ (entries 1 and 2). Among solvents tested, methanol was most suitable for this reaction (entries 1, 3, and 4). Although the counter cation of electrolytes had little effect on both yield and selectivity for the electrochemical reaction, $\mathrm{Et}_{4} \mathrm{NBr}$ was slightly better than other bromide salts (entries 1 and 5-7). On the other hand, use of either $\mathrm{Et}_{4} \mathrm{NCl}$ or $\mathrm{Et}_{4} \mathrm{NI}$ did not yield the oxidation product (entries 8 and 9). 
$-[\mathrm{e}]$<smiles>OC1CCCC[C@@]1(O)c1ccccc1</smiles>

1

$0.5 \mathrm{mmol}$
Pt electrodes, $2 \mathrm{~F} / \mathrm{mol}, 0^{\circ} \mathrm{C}$ electrolyte (1.0 equiv) solvent $(5 \mathrm{~mL})$

$\mathrm{Cu}(\mathrm{OTf})_{2}$ (0.1 equiv)

$(R, R)$-Ph-BOX (0.1 equiv)<smiles>O=C1CCCC[C@]1(O)c1ccccc1</smiles>

(S)-2<smiles>OC1CCCCC1(O)c1ccccc1</smiles>

$(R, R)-1$

Table 1. Effect of electrolyte and solvent on electrochemical oxidation of 1

\begin{tabular}{|c|c|c|c|c|c|c|c|}
\hline \multirow{2}{*}{ entry } & \multirow{2}{*}{ electrolyte } & \multirow{2}{*}{ solvent } & \multicolumn{2}{|c|}{ (S)-2 } & \multicolumn{2}{|c|}{$(R, R)-1$} & \multirow{2}{*}{$S$} \\
\hline & & & yield (\%) & ee $(\%)$ & yield (\%) & ee (\%) & \\
\hline 1 & $\mathrm{Et}_{4} \mathrm{NBr}$ & $\mathrm{MeOH}$ & 36 & 67 & 58 & 41 & 8 \\
\hline $2^{\mathrm{a}}$ & $\mathrm{Et}_{4} \mathrm{NBr}$ & $\mathrm{MeOH}$ & 33 & 66 & 62 & 36 & 7 \\
\hline 3 & $\mathrm{Et}_{4} \mathrm{NBr}$ & $\mathrm{MeCN}$ & trace & & 91 & - & - \\
\hline 4 & $\mathrm{Et}_{4} \mathrm{NBr}$ & $\mathrm{CH}_{2} \mathrm{Cl}_{2}$ & 8 & 13 & 79 & 2 & 1 \\
\hline 5 & $\mathrm{Me}_{4} \mathrm{NBr}$ & $\mathrm{MeOH}$ & 28 & 60 & 67 & 28 & 5 \\
\hline 6 & $n-\mathrm{Pr}_{4} \mathrm{NBr}$ & $\mathrm{MeOH}$ & 26 & 67 & 62 & 33 & 7 \\
\hline 7 & $n-\mathrm{Bu}_{4} \mathrm{NBr}$ & $\mathrm{MeOH}$ & 31 & 59 & 63 & 41 & 6 \\
\hline 8 & $\mathrm{Et}_{4} \mathrm{NCl}$ & $\mathrm{MeOH}$ & trace & - & 94 & - & - \\
\hline 9 & $\mathrm{Et}_{4} \mathrm{NI}$ & $\mathrm{MeOH}$ & 0 & - & quant & - & - \\
\hline
\end{tabular}

${ }^{\mathrm{a}} \mathrm{CuBr}_{2}$ (0.1 equiv) was used instead of $\mathrm{Cu}(\mathrm{OTf})_{2}$ ( 0.1 equiv).

\subsection{Asymmetric oxidation of several cis-cycloalkane-1,2-diols}

Asymmetric electrochemical oxidation of several cis-cycloalkane-1,2-diol derivatives 3-8 are summarized in Table 2 (Eq. 5). ${ }^{10}$ The $s$ values of (S)-9-11 varied significantly depending on the ring size (entries 1-3 in Table 2 and entry1 in Table 1). That is, the larger the ring size, the better $s$ value obtained. R substituent also influenced the $s$ value (entries 4-6). Compound 8 with a cyclohexyl group was asymmetrically oxidized to afford $(S)-\mathbf{1 4}$ in higher selectivity ( $(=11$, entry 6$)$ than $(S)-\mathbf{1 2}$ with a benzyl group (s=2, entry 6$)$ and (S)-13 with an isopropyl group (s=9, entry 5). 
<smiles>[R]C1(O)CCCC1O</smiles>

3-8 $-[\mathrm{e}]$

Pt electrodes, $2 \mathrm{~F} / \mathrm{mol}, 0^{\circ} \mathrm{C}$ $\underset{\mathrm{MeOH}}{\mathrm{Et}} \mathrm{NBr}(1.0$ equiv) $\mathrm{Cu}(\mathrm{OTf})_{2}$ (0.1 equiv) $(R, R)-\mathrm{Ph}-\mathrm{BOX}$ (0.1 equiv)<smiles>[R][C@]1(O)CCCC1=O</smiles>

$(S)-9-14$<smiles>[R]C1CCCC1O</smiles>

$(R, R)-3-8$

Table 2. Electrochemical oxidation of cis-cycloalkane-1,2-diols 3-8

\begin{tabular}{|c|c|c|c|c|c|c|c|c|c|}
\hline \multirow{2}{*}{ entry } & \multirow{2}{*}{ diol } & \multirow{2}{*}{$\mathrm{n}$} & \multirow{2}{*}{$\mathrm{R}$} & & \multicolumn{2}{|c|}{ (S)-ketoalocohol } & \multicolumn{2}{|c|}{$(R, R)$-diol } & \multirow{2}{*}{$s$} \\
\hline & & & & & yield (\%) & ee $(\%)$ & yield (\%) & ee (\%) & \\
\hline 1 & 3 & 1 & $\mathrm{Ph}$ & 9 & 33 & 38 & 59 & 22 & 3 \\
\hline 2 & 4 & 3 & $\mathrm{Ph}$ & 10 & 57 & 64 & 42 & 85 & 12 \\
\hline 3 & 5 & 4 & $\mathrm{Ph}$ & 11 & 49 & 80 & 48 & 80 & 22 \\
\hline 4 & 6 & 2 & $\mathrm{Bn}$ & 12 & 65 & 5 & 35 & 47 & 2 \\
\hline 5 & 7 & 2 & $i-\operatorname{Pr}$ & 13 & 26 & 75 & 74 & 25 & 9 \\
\hline 6 & 8 & 2 & Cyclohexyl & 14 & 21 & 81 & 76 & 18 & 11 \\
\hline
\end{tabular}

\subsection{Oxidative kinetic resolution of piperidine-3,4-diols}

This electrochemical method, interestingly, was appliable to kinetic resolution of some piperidine-3,4-diols 15, 18, and 22 (Eqs. 6-8), ${ }^{11}$ which were not oxidized with NBS. $^{3}$ The electrochemical oxidation of $\mathbf{1 5}$ afforded optically active ketoalcohol $(R)-\mathbf{1 7}$ and (3S,4R)-15 in 49\% ee and 41\% ee, respectively, while 16 was not obtained (Eq. 6). The loss in the total yield (82\%) in the reaction of $\mathbf{1 5}$ might be explained by instability of 3-ketopiperidines under the oxidative conditions. ${ }^{12}$ In fact, the oxidation of $\mathbf{1 8}$ afforded $(3 S, 4 S)-18$ in moderate enantioselectivity along with a small amount of further oxidized products 20 and 21 instead of 19 (Eq. 7). On the other hand, oxidation of 22 gave $(R)$-23and (3S,4R)-22 with little loss in the total yield (Eq. 8). 

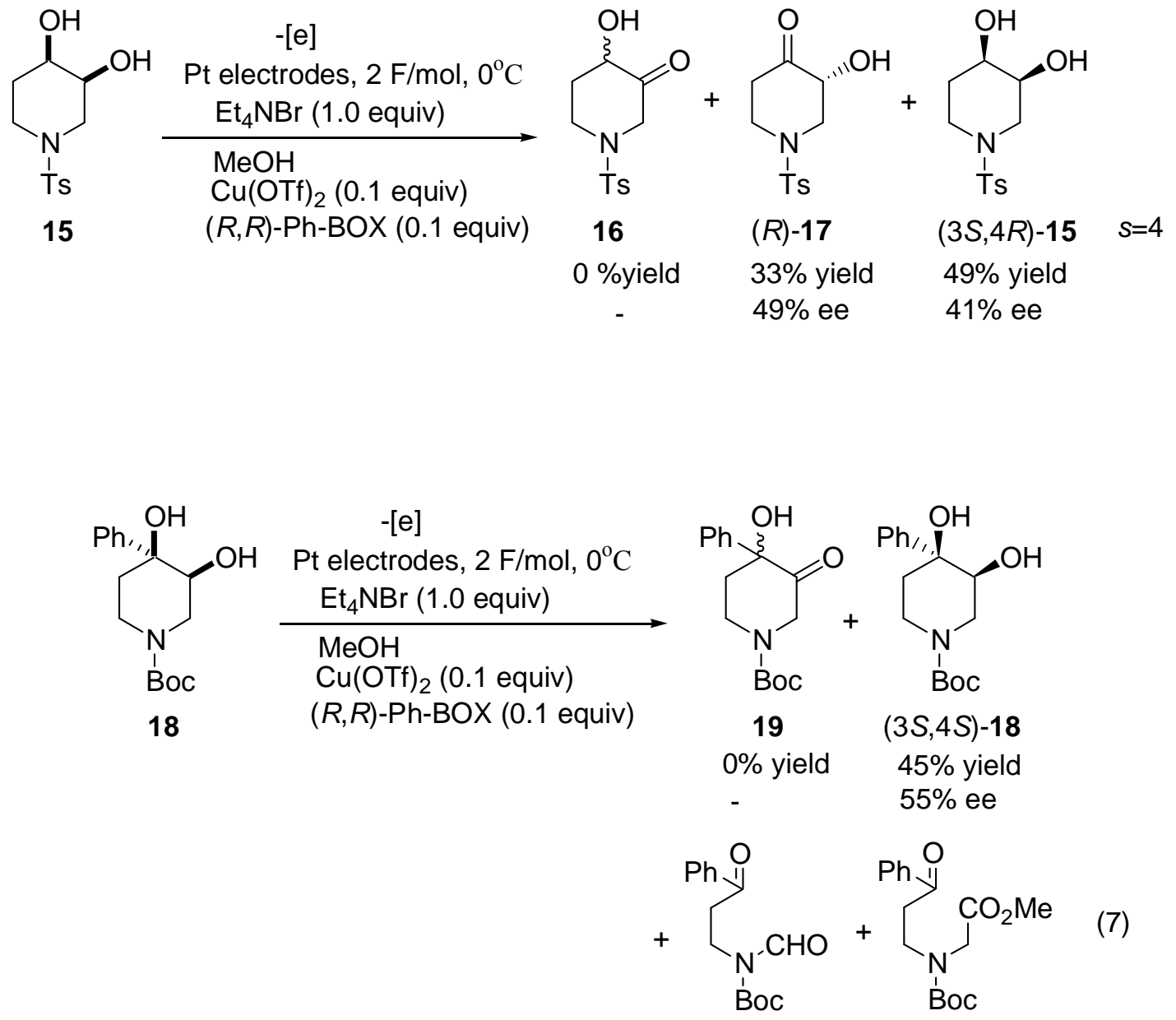

20

21

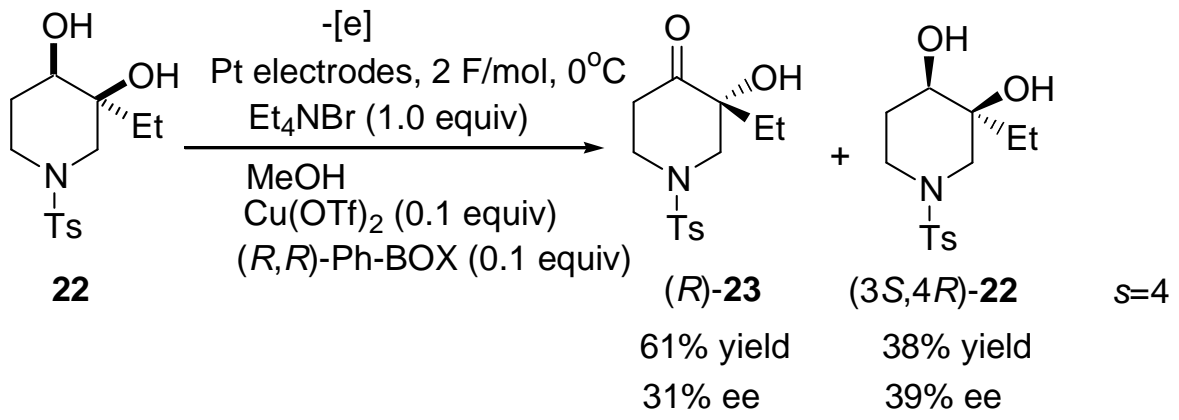

\subsection{Oxidative kinetic resolution of racemic aminoalcohols and aminoaldehydes}

Kinetic resolution of some racemic aminoalcohols 24-27 by electrochemical oxidation was tested (Eq. 9). The results are summarized in Table 3. In the oxidation of 
racemic- $N$-benzoylpiperidinemethanol (24), ${ }^{13}$ optically active $\alpha$-aminoester $(R)$-28 was obtained with low yield but good enantioselectivity (6\% yield, $71 \%$ ee) after passing through $5 \mathrm{~F} / \mathrm{mol}^{14}$ of electricity (entry 1). The oxidation of racemic $\mathrm{N}$-benzoylated cyclic and acyclic aminoalcohols 25-27 afforded the corresponding $(R)$-aminoesters (R)-29-31 (entries 2-4) with low electron efficiencies.

-[e]

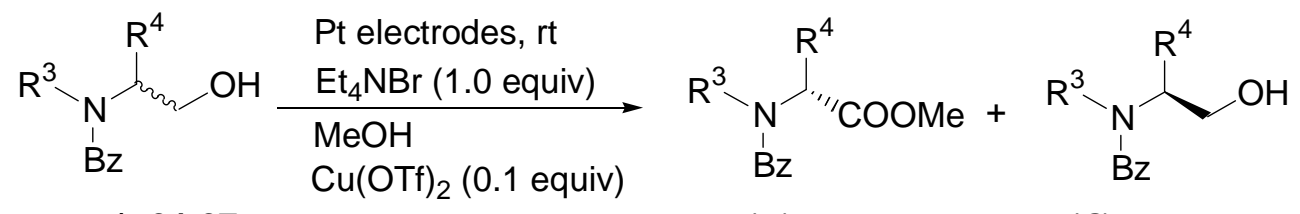

$\begin{array}{llll}\text { racemic-24-27 } & (R, R) \text {-Ph-BOX (0.1 equiv) } & (R)-28-31 & (S)-24-27\end{array}$

Table 3. Electrochemical oxidation of racemic aminoalcohols 24-27

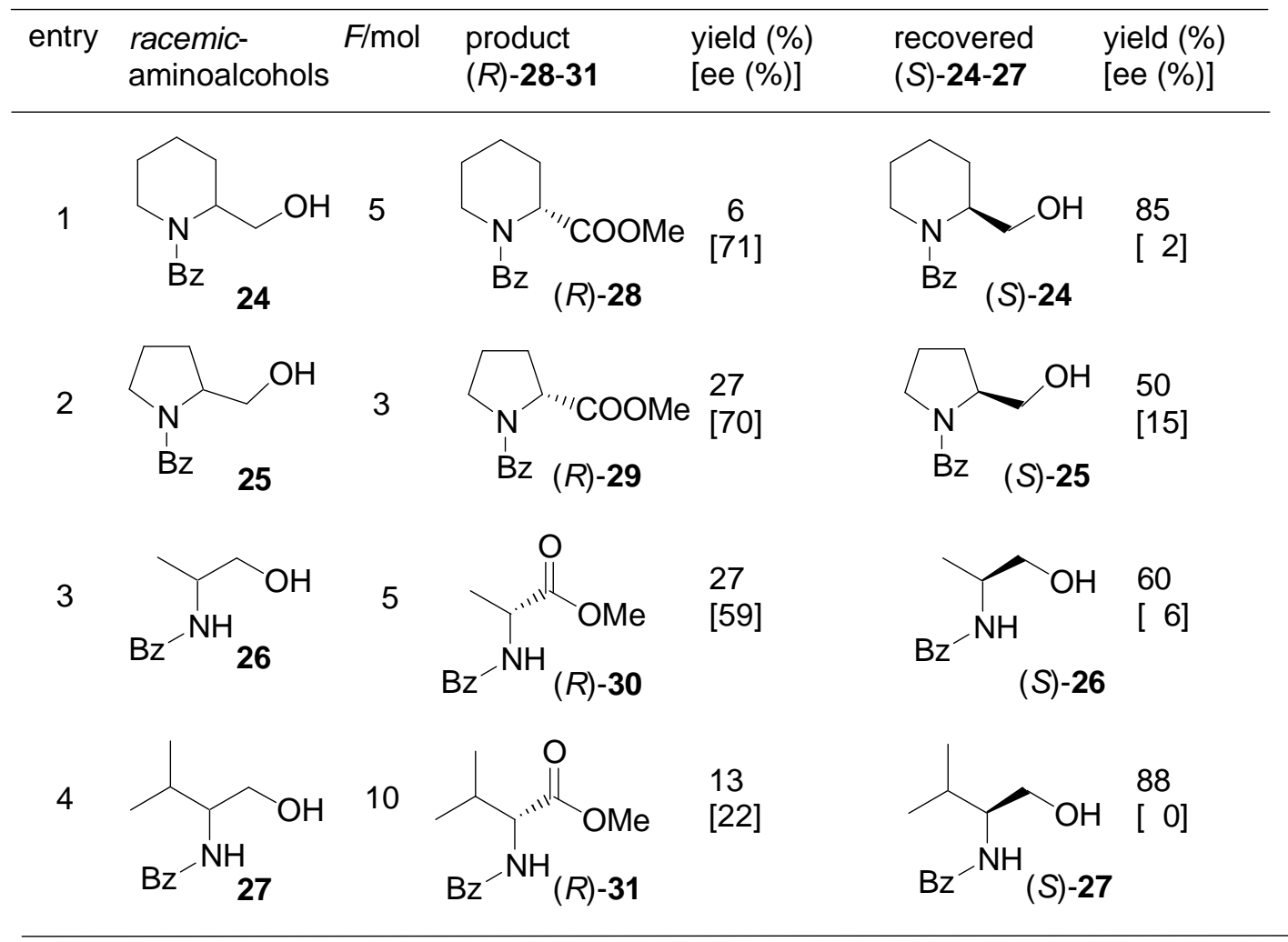

Since further improvement of the electron efficiency and yield was difficult, we 
tried oxidative kinetic resolution of racemic $N$-benzoylaminoaldehyde (32-35) (Eq. 10), which might be intermediates for the oxidation of aminoalcohols to amino esters. The results are shown in Table 4 . The oxidation of 32 proceeded more efficiently than that of aminoalcohol 24 to afford $(R)-\mathbf{2 8}$ with improved yield and good enantioselectivity after passing through $2.5 \mathrm{~F} / \mathrm{mol}^{14}$ of electricity (entry 1). Also, the oxidation of several aminoaldehydes (33-35) proceeded to afford $(R)$-29-31 with improved yields and good enantioselectivities (entries 2-4).

-[e]

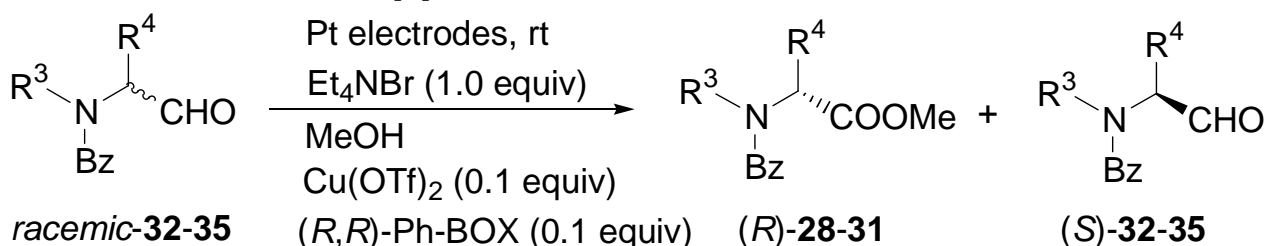

Table 4. Electrochemical oxidation of racemic aminoaldehydes 32-35

\begin{tabular}{|c|c|c|c|c|c|c|}
\hline entry & $\begin{array}{l}\text { racemic- } \\
\text { aminoaldehydes }\end{array}$ & F/mol & $\begin{array}{l}(R) \text {-amino- } \\
\text { esters }\end{array}$ & $\begin{array}{l}\text { yield (\%) } \\
\text { [ee (\%)] }\end{array}$ & $\begin{array}{l}\text { (S)-amino- } \\
\text { aldehydes }\end{array}$ & $\begin{array}{l}\text { yield }(\%) \\
{[\text { ee }(\%)]^{a}}\end{array}$ \\
\hline 1 & $\mathrm{CHO}$ & 2.5 & $(R)-28$ & $\begin{array}{l}19 \\
{[87]}\end{array}$ & $(S)-32$ & $\begin{array}{l}66 \\
{[12]}\end{array}$ \\
\hline & 32 & & & & & \\
\hline 2 & 33 & 2 & $(R)-29$ & $\begin{array}{l}43 \\
{[86]}\end{array}$ & $(S)-33$ & $\begin{array}{l}34 \\
{[27]}\end{array}$ \\
\hline 3 & $\mathrm{Bz}^{\prime}$ & 1.5 & $(R)-30$ & $\begin{array}{l}50 \\
{[61]}\end{array}$ & $(S)-34$ & $\begin{array}{l}18 \\
{[18]}\end{array}$ \\
\hline 4 & $\mathrm{Bz}^{-\mathrm{NH}} 35$ & 1.5 & $(R)-31$ & $\begin{array}{l}18 \\
{[64]}\end{array}$ & $(S)-35$ & $\begin{array}{l}65 \\
{[12]}\end{array}$ \\
\hline
\end{tabular}

a Determined after transformation of (S)-32-35 to (S)-24-27, respectively. 


\subsection{Oxidation potentials}

Oxidation potentials of materials used in these oxidations are shown in Table 5. The most oxidizable species was $\mathrm{Br}^{-}$, while $\mathrm{Cu}(\mathrm{OTf})_{2}$ was hardly oxidizable (entries 1 and 2). Although oxidation potential of $(R, R)-\mathrm{Ph}-\mathrm{BOX}$ was moderate, $\mathrm{Cu}(\mathrm{OTf})_{2}-(R, R)-\mathrm{Ph}-\mathrm{BOX}$ complex was stable under the oxidative conditions (entries 3 and 4). Diol cis-1 formed a complex with $\mathrm{Cu}(\mathrm{OTf})_{2}-(R, R)-\mathrm{Ph}-\mathrm{BOX}$ which was more oxidizable (entries 5 and 6). Similar tendencies were observed for aminoalcohol 24 and aminoaldehyde 32(entries 9-12). On the other hand, such negative shifts were not observed in the oxidation potentials of ketoalcohol 2 and aminoester 28 (entries 7, 8, 13, and 14). Among these complexes, cis-1-Cu(OTf $)_{2}-(R, R)-\mathrm{Ph}-\mathrm{BOX}$ and 32-Cu(OTf $)_{2}-(R, R)-\mathrm{Ph}-\mathrm{BOX}$ complexes had the lowest oxidation potentials (entries 6 and 12).

Table 5. Oxidation potentials

\begin{tabular}{lll}
\hline entry & material & oxidation potential $(\mathrm{V})^{\mathrm{a}}$ \\
\hline 1 & $\mathrm{Et}_{4} \mathrm{NBr}$ & 1.15 \\
2 & $\mathrm{Cu}(\mathrm{OTf})_{2}$ & $>3.0$ \\
3 & $(R, R)-\mathrm{Ph}-\mathrm{BOX}$ & 2.07 \\
4 & $\mathrm{Cu}(\mathrm{OTf})_{2}-(R, R)-\mathrm{Ph}-\mathrm{BOX}$ complex & $>3.0$ \\
5 & diol cis-1 & 2.10 \\
6 & cis-1-Cu$(\mathrm{OTf})_{2}-(R, R)-\mathrm{Ph}-\mathrm{BOX}$ complex & 1.80 \\
7 & ketoalcohol 2 & 2.20 \\
8 & 2-Cu(OTf $)_{2}-(R, R)-\mathrm{Ph}-\mathrm{BOX}$ complex & 2.35 \\
9 & aminoalcohol 24 & 2.20 \\
10 & 24-Cu(OTf $)_{2}-(R, R)-\mathrm{Ph}-\mathrm{BOX}$ complex & 2.10 \\
11 & aminoaldehyde 32 & 2.10 \\
12 & 32-Cu$(\mathrm{OTf})_{2}-(R, R)-\mathrm{Ph}-\mathrm{BOX}$ complex & 1.75 \\
13 & aminoester 28 & 2.32 \\
14 & 28-Cu(OTf $)_{2}-(R, R)-\mathrm{Ph}-\mathrm{BOX}$ complex & 2.65 \\
\hline
\end{tabular}

${ }^{\mathrm{a}}$ V vs. $\mathrm{Ag} / \mathrm{AgNO}_{3}$ 
2.7 Reaction mechanism for the asymmetric electrochemical oxidation of aminoalcohols and aminoaldehydes

On the basis of oxidation potentials described above, mechanisms for asymmetric electrochemical oxidation of aminoalcohols G' and aminoaldehydes $\mathbf{H}^{\prime}$ are presented in Schemes 2 and 3, respectively. The electrochemical oxidation of $\mathbf{G}^{\prime}$ and $\mathbf{H}^{\prime}$ might proceed in similar manner to that of diols as shown in Scheme 1. Anodically generated $\mathrm{Br}^{+}$and cathodically generated $2 \mathrm{MeO}^{-}$react with complexes $\mathbf{I}^{\prime}$ and/or $\mathbf{M}^{\prime}$ to afford corresponding optically active esters $\mathbf{P}$ '.

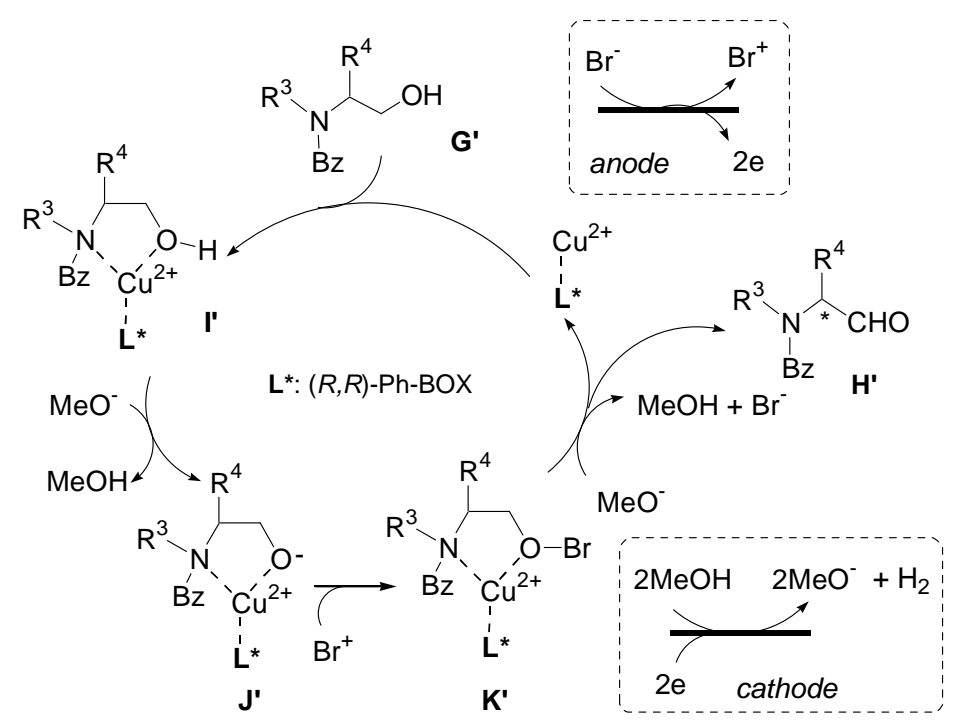

Scheme 2 


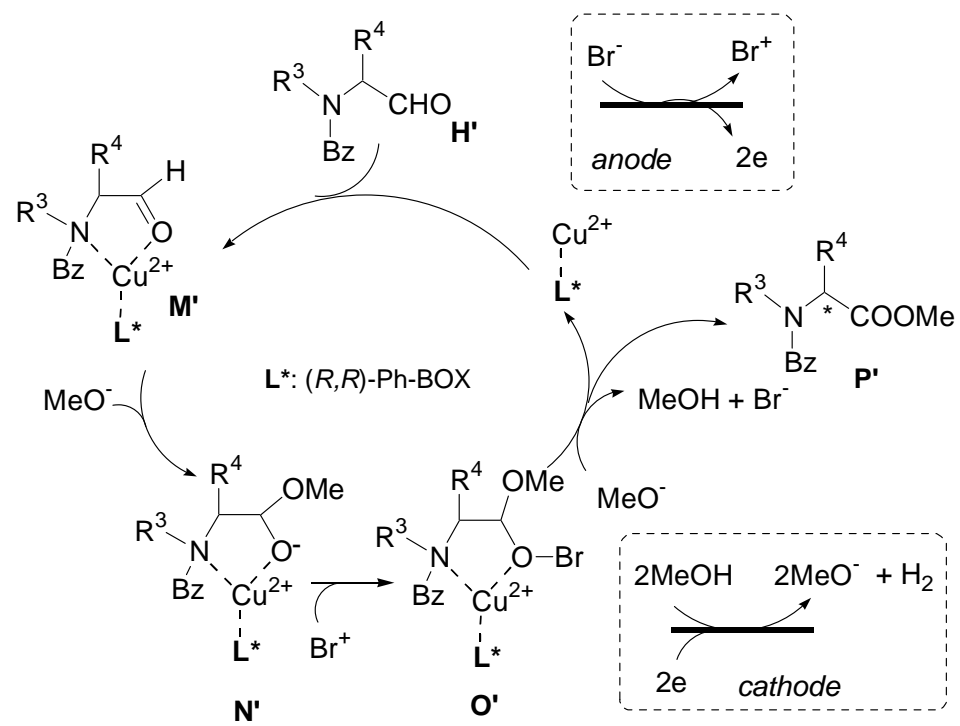

Scheme 3

The stereochemical courses for kinetic resolution of cis-1 and $\mathbf{3 2}$ catalyzed by $\mathrm{Cu}(\mathrm{II})-(R, R)-\mathrm{Ph}-\mathrm{BOX}$ are shown in Schemes 4 and 5. Although the activated intermediates $(R, R)$-1-complex and $(S)$-32-complex might be formed more easily than $(S, S)$-1-complex and $(R)$-32-complex, $\mathrm{MeO}^{-}$as a base predominantly approaches the less crowded intermediate $(S, S)$-1-complex and $(R)$-32-complex to afford $(S)-2$ and (R)-28. 


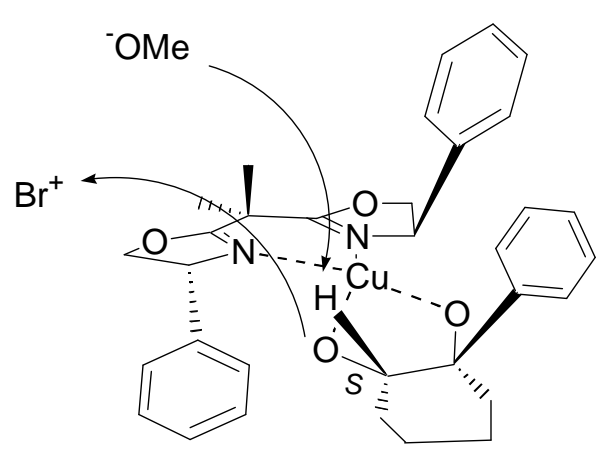

$(S, S)$-1-complex

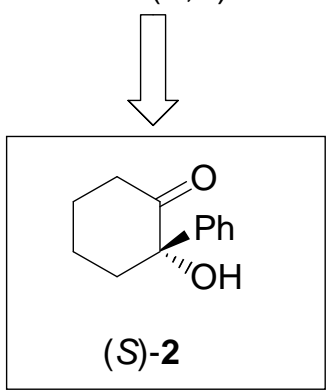

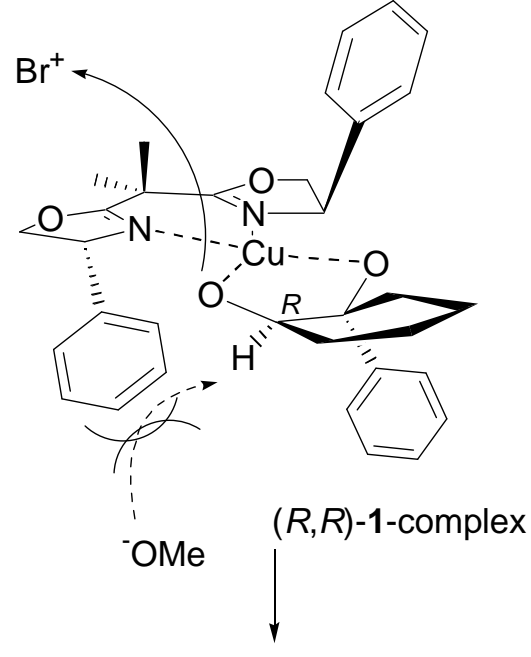

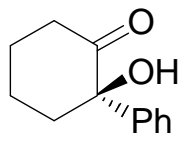

$(R)-2$

Scheme 4. Plausible stereochemical course for kinetic resolution of cis-1.

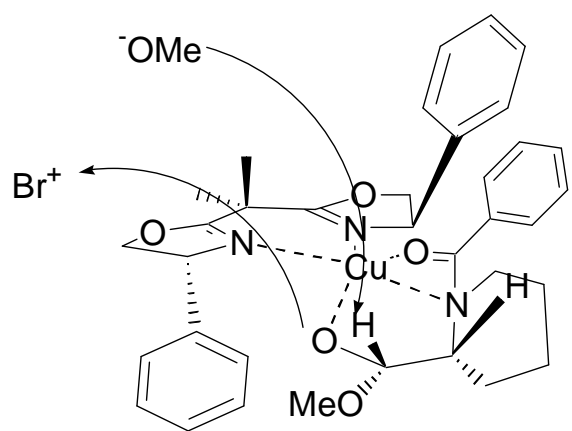

(R)-32-complex

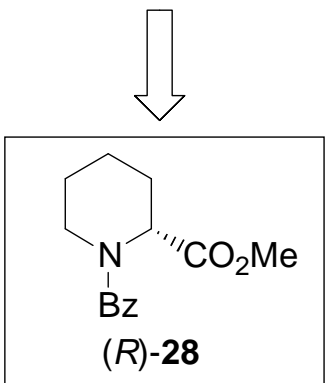

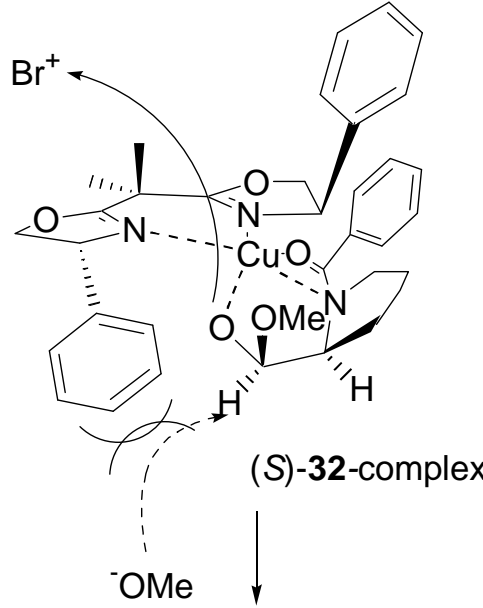

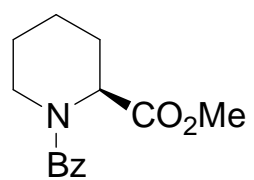

(S)-28

Scheme 5. Plausible stereochemical course for kinetic resolution of racemic-32 in $\mathrm{MeOH}$. 


\section{Conclusion}

In conclusion, this article describes an efficient procedure for the kinetic resolution of racemic cis-cycloalkane-1,2-diols, aminoalcohols and aminoaldehydes in the presence of copper(II) triflate and $(R, R)-\mathrm{Ph}-\mathrm{BOX}$ by electrochemical oxidation. Further study to improve the enantioselectivity is underway.

\section{Experimental Section}

\subsection{General}

Electrochemical reactions were carried out by using DC Power Supply (GP 050-2) of Takasago Seisakusho, Inc. HPLC analyses were achieved by using a LC-10AT VP and a SPD-10A VP of Shimadzu Seisakusho Inc. Specific rotations were measured with Jasco DIP-1000. ${ }^{1} \mathrm{H}$ and ${ }^{13} \mathrm{CNMR}$ spectra were measured at 300 (or 400) and 75 (or 100) $\mathrm{MHz}$ on a Varian Gemini 300 spectrometer (or JEOL JNM-AL 400) with TMS as an internal standard. All melting points were measured on MICRO MELTING POINT APPARATUS (Yanaco) and are uncorrected. IR spectra were obtained on a Shimadzu FTIR-8100A. Mass spectra were obtained on a JEOL JMS-DX 303 instrument.

$(R, R)-\mathrm{Ph}-\mathrm{BOX}, \mathrm{Cu}(\mathrm{OTf})_{2}, \mathrm{Me}_{4} \mathrm{NBr}, \mathrm{Et}_{4} \mathrm{NBr}, n-\mathrm{Pr}_{4} \mathrm{NBr}, n-\mathrm{Bu}_{4} \mathrm{NBr}, \mathrm{Et}_{4} \mathrm{NCl}$, and $\mathrm{Et}_{4} \mathrm{NI}$ were commercially available.

\subsection{Measurement of oxidation potentials}

BAS CV-50W was used as a voltametric analyzer. A solution of substrate $(0.1$ mmol) in $\mathrm{MeCN}(10 \mathrm{~mL})$ containing $0.1 \mathrm{M} \mathrm{Et}_{4} \mathrm{NBF}_{4}$ was measured. Reference electrode was $\mathrm{Ag} / \mathrm{AgNO}_{3}$ in saturated aqueous $\mathrm{KCl}$, a working electrode was a glassy carbon, and a counter electrode was a platinum wire. Scan rate was $100 \mathrm{mV} / \mathrm{s}$. 


\subsection{Preparation of cis-cycloalkane-1,2-diols 3-8}

$4 \% \mathrm{OsO}_{4}(0.098 \mathrm{mmol})$ was added to a stirring solution of 1-phenylcyclohexene (32 mmol, $5.0 \mathrm{~g}$ ) and 50\% $\mathrm{N}$-methylmorpholine- $\mathrm{N}$-oxide (35.4 mmol) in THF-acetone- $\mathrm{H}_{2} \mathrm{O}$ $(1: 1: 1,180 \mathrm{~mL})$ at $0^{\circ} \mathrm{C}$. After stirring for $16 \mathrm{~h}$, sat. $\mathrm{NaHSO}_{3}$ aqueous $(120 \mathrm{~mL})$ was added the resulting mixture and the solution stirred for $1 \mathrm{~h}$. The solution was evaporated in vacuo to give residue, which was then dissolved in AcOEt. The organic portion was extracted with AcOEt and dried over $\mathrm{MgSO}_{4}$. The resulting solution was concentrated in vacuo. The residue was chromatographed on silica gel (hexane/AcOEt $=5 / 1$ ) to afford 1 (93\% yield).

\subsection{Asymmetric electrochemical oxidation of 1: a typical procedure}

Under an aerobic atmosphere, into an undivided electrolysis cell equipped with a platinum anode and cathode $(1 \mathrm{~cm} \times 2 \mathrm{~cm})$ was added a solution of $\mathrm{Cu}(\mathrm{OTf})_{2}(18.1 \mathrm{mg}$, $0.05 \mathrm{mmol})$ and $(R, R)-\mathrm{Ph}-\mathrm{BOX}(16.7 \mathrm{mg}, 0.05 \mathrm{mmol})$ in $\mathrm{MeOH}(5 \mathrm{~mL})$. This solution was stirred for $10 \mathrm{~min}$. Into the solution were added 1-phenylcyclohexane-cis-1,2-diol (1, $96.1 \mathrm{mg}, 0.5 \mathrm{mmol})$, tetraethylammonium bromide (105.1 $\mathrm{mg}, 0.5 \mathrm{mmol})$, and 2 $\mathrm{F} / \mathrm{mol}$ of electricity was passed at a constant rate of $50 \mathrm{~mA}$ (terminal voltage; ca. $10 \mathrm{~V}$ ) through the solution as it was cooled with ice-water. The solvent was evaporated in vacuo to give residue, which was then dissolved in AcOEt. The solution was poured into sat. aqueous $\mathrm{Na}_{2} \mathrm{~S}_{2} \mathrm{O}_{3}(5 \mathrm{~mL})$. The organic portion was extracted with AcOEt $(10 \mathrm{~mL} \mathrm{x}$ 3) and dried over $\mathrm{MgSO}_{4}$. The resulting solution was concentrated in vacuo. The residue was purified by silica gel column chromatography ( $n$-hexane:AcOEt $=10: 1$ ) to afford (S)-2-hydroxy-2-phenylcyclohexanone (2) as a white solid and recovered 
$(1 R, 2 R)-1$-phenylcyclohexane-cis-1,2-diol (1) as a white solid.

\subsubsection{1 (S)-2-Hydroxy-2-phenylcyclohexanone (2)}

White solid; $\mathrm{mp} 74^{\circ} \mathrm{C} ;[\alpha]_{\mathrm{D}}^{29}+119.3\left(c=1.2, \mathrm{CHCl}_{3}, 67 \%\right.$ ee); IR (neat) v 3474, 2944, 2867, 1717, 1451, $1101 \mathrm{~cm}^{-1} ;{ }^{1} \mathrm{H}$ NMR $\left(300 \mathrm{MHz}, \mathrm{CDCl}_{3}\right) \delta 1.62-1.95(\mathrm{~m}, 4 \mathrm{H})$, 2.00-2.10 (m, 1H), 2.38-2.60 (m, 2H), 2.95-3.05 (m, 1H), $4.50(\mathrm{~s}, 1 \mathrm{H}), 7.26-7.42(\mathrm{~m}$, 5H); HR-EI $\left(\mathrm{M}^{+}\right)$calcd for $\mathrm{C}_{12} \mathrm{H}_{14} \mathrm{O}_{2} 190.0994$ found 190.0972 .

The ee was determined by DAICEL Chiralcel OJ-H (4.6 $\mathrm{mm} \phi, 250 \mathrm{~mm})$ [n-hexane/isopropanol (10/1) (v/v), $1.0 \mathrm{~mL} / \mathrm{min}$, detection at $210 \mathrm{~nm}, 15 \mathrm{~min}$ for $(R)-2$ and $19 \min$ for $(S)-2]$.

4.4.1.2 (1R,2R)-1-Phenylcyclohexane-cis-1,2-diol (1) $)^{15}$

White solid; mp $90^{\circ} \mathrm{C}$ (lit. $\left.{ }^{15} \mathrm{mp} 121-122^{\circ} \mathrm{C}\right) ;[\alpha]_{\mathrm{D}}{ }^{25}-3.7\left(c=1.2\right.$, EtOH, $41 \%$ ee); ${ }^{1} \mathrm{H}$ NMR $\left(300 \mathrm{MHz}, \mathrm{CDCl}_{3}\right) \delta 1.35-1.95(\mathrm{~m}, 9 \mathrm{H}), 2.62(\mathrm{~s}, 1 \mathrm{H}), 3.92-4.04(\mathrm{~m}, 1 \mathrm{H})$, 7.22-7.28 (m, 1H), $7.38(\mathrm{t}, J=7.8 \mathrm{~Hz}, 2 \mathrm{H}), 7.51(\mathrm{~d}, J=7.2 \mathrm{~Hz}, 2 \mathrm{H})$. The ee was determined by DAICEL Chiralcel OJ-H (4.6mm $\phi, 250 \mathrm{~mm})$ [n-hexane/isopropanol (10/1) (v/v), 1.0 $\mathrm{mL} / \mathrm{min}$, detection at $210 \mathrm{~nm}, 10 \mathrm{~min}$ for $(1 S, 2 S)-\mathbf{1}$ and $13 \mathrm{~min}$ for $(1 R, 2 R)-\mathbf{1}]$.

\subsubsection{1 (S)-2-Hydroxy-2-phenylcyclopentanone (9)}

Light-yellow oil (lit. ${ }^{16}$ for racemate, $\left.\operatorname{mp~} 67^{\circ} \mathrm{C}\right) ;[\alpha]_{\mathrm{D}}{ }^{18}+57.4\left(c=1.9, \mathrm{CHCl}_{3}, 38 \%\right.$ ee); ${ }^{1} \mathrm{H}$ NMR $\left(300 \mathrm{MHz}, \mathrm{CDCl}_{3}\right) \delta 1.79-1.94(\mathrm{~m}, 1 \mathrm{H}), 2.00-2.16(\mathrm{~m}, 1 \mathrm{H}), 2.16-2.31(\mathrm{~m}, 1 \mathrm{H})$, 2.40-2.58 (m, 3H), $2.88(\mathrm{~s}, 1 \mathrm{H}), 7.27-7.46(\mathrm{~m}, 5 \mathrm{H})$. The ee was determined by DAICEL Chiralcel OJ-H (4.6 mm $\phi, 250 \mathrm{~mm})[n$-hexane/isopropanol $(10 / 1)(\mathrm{v} / \mathrm{v}), 1.0 \mathrm{~mL} / \mathrm{min}$, detection at $210 \mathrm{~nm}, 16 \mathrm{~min}$ for $(R)-9$ and $20 \min$ for $(S)-9]$. 
4.4.2.2 (1R,2R)-1-Phenylcyclopentane-cis-1,2-diol (3) (lit. ${ }^{17}$ for racemate)

White solid; mp $62-63^{\circ} \mathrm{C} ; \quad[\alpha]_{\mathrm{D}}^{29}-7.2 \quad\left(c=3.2, \quad \mathrm{CHCl}_{3}, 22 \%\right.$ ee); IR (neat) v 3400, 2967, 1447, 1092, 1055, 758, $700 \mathrm{~cm}^{-1} ;{ }^{1} \mathrm{H}$ NMR $\left(300 \mathrm{MHz}, \mathrm{CDCl}_{3}\right) \delta 1.60-1.86$ (m, 2H), 1.86-2.16 (m, 4H), 2.30 (br s, 1H), 2.80 (br s, $1 \mathrm{H}), 4.22$ (t, J=7.5Hz, 1H), 7.15-7.28 (m, 1H), $7.33(\mathrm{t}, J=7.5 \mathrm{~Hz}, 2 \mathrm{H}), 7.45(\mathrm{~d}, 2 \mathrm{H})$. The ee was determined by DAICEL Chiralcel OJ-H (4.6 mm $\phi, 250 \mathrm{~mm})$ [n-hexane/isopropanol (10/1) (v/v), 1.0 $\mathrm{mL} / \mathrm{min}$, detection at $210 \mathrm{~nm}, 15 \mathrm{~min}$ for $(1 S, 2 S)-3$ and $19 \min$ for $(1 R, 2 R)-3]$.

\subsubsection{1 (S)-2-Hydroxy-2-phenylcycloheptanone (10) (lit. ${ }^{18}$ for racemate)}

Light-yellow oil; $[\alpha]_{\mathrm{D}}{ }^{29}+123.4\left(c=1.7, \mathrm{CHCl}_{3}, 64 \%\right.$ ee); ${ }^{1} \mathrm{H}$ NMR $\left(300 \mathrm{MHz}, \mathrm{CDCl}_{3}\right)$ $\delta 1.22-1.68(\mathrm{~m}, 3 \mathrm{H}), 1.83-2.10(\mathrm{~m}, 3 \mathrm{H}), 2.22-2.40(\mathrm{~m}, 2 \mathrm{H}), 2.40-2.55(\mathrm{~m}, 1 \mathrm{H})$, 2.75-2.88 (m, 1H), $4.58(\mathrm{~s}, 1 \mathrm{H}), 7.25-7.40(\mathrm{~m}, 3 \mathrm{H}), 7.40-7.50(\mathrm{~m}, 2 \mathrm{H})$. The ee was determined by DAICEL Chiralcel OJ-H (4.6 mm $\phi, 250 \mathrm{~mm})$ [ $n$-hexane/isopropanol $(10 / 1)(\mathrm{v} / \mathrm{v}), 1.0 \mathrm{~mL} / \mathrm{min}$, detection at $210 \mathrm{~nm}, 20 \mathrm{~min}$ for $(S)-\mathbf{1 0}$ and $30 \mathrm{~min}$ for $(R)-\mathbf{1 0}]$.

\subsubsection{2 (1R,2R)-1-Phenylcycloheptane-cis-1,2-diol (4)}

White solid; mp $99-100^{\circ} \mathrm{C} ;[\alpha]_{\mathrm{D}}{ }^{28}-1.9\left(c=1.0, \mathrm{CHCl}_{3}, 85 \%\right.$ ee) ; IR (neat) v 3400, 3023, 2930, 1460, $1019 \mathrm{~cm}^{-1} ;{ }^{1} \mathrm{H}$ NMR (400MHz, $\left.\mathrm{CDCl}_{3}\right) \delta 1.40-1.78(\mathrm{~m}, 7 \mathrm{H})$, 1.78-2.15 (m, 4H), $2.90(\mathrm{~s}, 1 \mathrm{H}), 4.00(\mathrm{~d}, J=10.4 \mathrm{~Hz}, 1 \mathrm{H}), 7.22-7.32(\mathrm{~m}, 1 \mathrm{H}), 7.32-7.47$ (m, 2H), 7.47-7.64 (m, 2H); ${ }^{13} \mathrm{C}$ NMR (100MHz, $\left.\mathrm{CDCl}_{3}\right) \delta$ 20.0, 22.7, 26.6, 29.8, 39.1, 77.5, 78.9, 124.8, 126.9, 128.5, 148.3; HR-EI $\left(\mathrm{M}^{+}\right)$calcd for $\mathrm{C}_{13} \mathrm{H}_{18} \mathrm{O}_{2} 206.1307$ found 206.1300. The ee was determined by DAICEL Chiralcel OJ-H (4.6 mm $\phi, 250 \mathrm{~mm})$ [n-hexane/isopropanol (10/1) (v/v), $1.0 \mathrm{~mL} / \mathrm{min}$, detection at $210 \mathrm{~nm}, 11 \mathrm{~min}$ for $(1 S$, 2S)-4 and $13 \mathrm{~min}$ for (1R, 2R)-4]. 


\subsubsection{1 (S)-2-Hydroxy-2-phenylcyclooctanone (11)}

Colorless oil; $[\alpha]_{\mathrm{D}}^{29}+177.7\left(c=2.0, \mathrm{CHCl}_{3}, 80 \%\right.$ ee); IR (neat) v 3467, 2930, 2859, 1701, 1698, 1466, 1447, 1364, $1127 \mathrm{~cm}^{-1} ;{ }^{1} \mathrm{H}$ NMR $\left(300 \mathrm{MHz}, \mathrm{CDCl}_{3}\right) \delta$ 0.96-1.12 (m, $1 \mathrm{H}), 1.35-1.62(\mathrm{~m}, 2 \mathrm{H}), 1.62-2.04(\mathrm{~m}, 5 \mathrm{H}), 2.04-2.16(\mathrm{~m}, 1 \mathrm{H}), 2.16-2.28(\mathrm{~m}, 1 \mathrm{H})$, 2.70-2.88 (m, 1H), 2.88-3.02 (m, 1H), $4.81(\mathrm{~s}, 1 \mathrm{H}), 7.25-7.40(\mathrm{~m}, 3 \mathrm{H}), 7.48(\mathrm{~d}, J=6.9 \mathrm{~Hz}$, $2 \mathrm{H}) ;{ }^{13} \mathrm{C} \mathrm{NMR}\left(100 \mathrm{MHz}, \mathrm{CDCl}_{3}\right) \delta 22.6,24.2,25.3,30.1,31.3,36.0,81.0,126.0,127.9$, 128.5, 141.4, 216.6; HR-EI $\left(\mathrm{M}^{+}\right)$calcd for $\mathrm{C}_{14} \mathrm{H}_{18} \mathrm{O}_{2} 218.1307$ found 218.1302. The ee was determined by DAICEL Chiralcel OJ-H (4.6 $\mathrm{mm} \phi, 250 \mathrm{~mm})$ [n-hexane/isopropanol $(10 / 1)(\mathrm{v} / \mathrm{v}), 1.0 \mathrm{~mL} / \mathrm{min}$, detection at $210 \mathrm{~nm}, 20 \mathrm{~min}$ for $(S)-\mathbf{1 1}$ and $28 \mathrm{~min}$ for $(R)-\mathbf{1 1}]$.

\subsubsection{2 (1R,2R)-1-Phenylcyclooctane-cis-1,2-diol (5)}

White solid; mp $46-47^{\circ} \mathrm{C} ;[\alpha]_{\mathrm{D}}^{29}-8.3\left(c=1.2, \mathrm{CHCl}_{3}, 80 \%\right.$ ee); IR (neat) v 3400, 2923, 1601, 1447, 1028, $702 \mathrm{~cm}^{-1} ;{ }^{1} \mathrm{H}$ NMR $\left(300 \mathrm{MHz}, \mathrm{CDCl}_{3}\right) \delta 1.20-1.92(\mathrm{~m}, 11 \mathrm{H})$, 2.00-2.15 (m, 1H), 2.24-2.40 (m, 1H), $2.98(\mathrm{~s}, 1 \mathrm{H}), 4.40(\mathrm{dd}, J=3.0 \mathrm{~Hz}, 8.7 \mathrm{~Hz}, 1 \mathrm{H})$, $7.22-7.32(\mathrm{~m}, 1 \mathrm{H}), 7.39(\mathrm{t}, J=7.8 \mathrm{~Hz}, 2 \mathrm{H}), 7.54(\mathrm{~d}, J=6.9 \mathrm{~Hz}, 2 \mathrm{H}) ;{ }^{13} \mathrm{C}$ NMR $(100 \mathrm{MHz}$, $\left.\mathrm{CDCl}_{3}\right) \delta 21.3,24.2,26.8,28.1,30.8,36.5,75.6,78.3,125.4,127.0,128.4,146.4$ HR-EI $\left(\mathrm{M}^{+}\right)$calcd for $\mathrm{C}_{14} \mathrm{H}_{20} \mathrm{O}_{2} 220.1554$ found 220.1454 . The ee was determined by DAICEL Chiralpak AS (4.6 mm $\phi, 250 \mathrm{~mm})$ [ $n$-hexane/isopropanol (10/1) (v/v), 1.0 $\mathrm{mL} / \mathrm{min}$, detection at $210 \mathrm{~nm}, 7 \mathrm{~min}$ for $(1 S, 2 S)-5$ and $13 \mathrm{~min}$ for $(1 R, 2 R)-5]$.

\subsubsection{1 (S)-2-Hydroxy-2-benzylcyclohexanone (12) ${ }^{19}$}

White solid; mp $55^{\circ} \mathrm{C} ;[\alpha]_{\mathrm{D}}{ }^{29}+6.3\left(c=1.7, \mathrm{CHCl}_{3}, 5 \%\right.$ ee); ${ }^{1} \mathrm{H}$ NMR $\left(300 \mathrm{MHz}, \mathrm{CDCl}_{3}\right)$ $\delta 1.60-1.80(\mathrm{~m}, 2 \mathrm{H}), 1.80-1.95(\mathrm{~m}, 2 \mathrm{H}), 2.14-2.47(\mathrm{~m}, 2 \mathrm{H}), 2.50-2.60(\mathrm{~m}, 1 \mathrm{H}), 2.70(\mathrm{dt}$, 
$J=6.0 \mathrm{~Hz}, 13.5 \mathrm{~Hz}, 1 \mathrm{H}), 2.97$ (d, J=13.7Hz, 1H), $3.14(\mathrm{~d}, J=13.7 \mathrm{~Hz}, 1 \mathrm{H}), 3.85(\mathrm{~s}, 1 \mathrm{H})$, 7.18-7.36 (m, 5H). The ee was determined by DAICEL Chiralpak AD (4.6 mm $\phi, 250$ $\mathrm{mm})$ [n-hexane/isopropanol (10/1) (v/v), $1.0 \mathrm{~mL} / \mathrm{min}$, detection at $254 \mathrm{~nm}, 9 \mathrm{~min}$ for (S)-12 and $11 \min$ for $(R)-12]$.

\subsubsection{2 (1R,2R)-1-Benzylcyclohexane-cis-1,2-diol (6)}

White solid; mp $103-104^{\circ} \mathrm{C}\left(\right.$ lit. $^{20}$ for racemate, mp $\left.107-108^{\circ} \mathrm{C}\right) ;[\alpha]_{\mathrm{D}}{ }^{29}-0.3(c=1.0$, $\mathrm{CHCl}_{3}, 47 \%$ ee); IR (neat) v 3404, $2938 \mathrm{~cm}^{-1} ;{ }^{1} \mathrm{H}$ NMR $\left(300 \mathrm{MHz}, \mathrm{CDCl}_{3}\right) \delta 1.16-1.92$ (m, 8H), $2.85(\mathrm{~d}, J=13.5 \mathrm{~Hz}, 2 \mathrm{H}), 2.95(\mathrm{~d}, J=13.5 \mathrm{~Hz}, 2 \mathrm{H}), 3.40-3.50(\mathrm{~m}, 1 \mathrm{H}), 7.20-7.35$ $(\mathrm{m}, 5 \mathrm{H})$. The ee was determined by DAICEL Chiralcel OJ-H (4.6 mm $\phi, 250 \mathrm{~mm})$ [n-hexane/isopropanol (10/1) (v/v), $1.0 \mathrm{~mL} / \mathrm{min}$, detection at $210 \mathrm{~nm}, 9 \mathrm{~min}$ for $(1 S, 2 S)-6$ and $11 \mathrm{~min}$ for $(1 R, 2 R)-6]$.

4.4.6.1 (S)-2-Hydroxy-2-isopropylcyclohexanone (13) (lit. ${ }^{21}$ for racemate)

Colorless oil; $[\alpha]_{\mathrm{D}}{ }^{29}+44.1\left(c=0.4, \mathrm{CHCl}_{3}, 75 \%\right.$ ee $) ;{ }^{1} \mathrm{H}$ NMR $\left(300 \mathrm{MHz}, \mathrm{CDCl}_{3}\right) \delta 0.70$ $(\mathrm{d}, J=6.9 \mathrm{~Hz}, 3 \mathrm{H}), 1.01(\mathrm{~d}, J=6.6 \mathrm{~Hz}, 3 \mathrm{H}), 1.55-1.80(\mathrm{~m}, 4 \mathrm{H}), 2.06-2.18(\mathrm{~m}, 1 \mathrm{H})$, 2.18-2.32 (m, 1H), 2.32-2.43 (m, 1H), 2.43-2.53 (m, 2H), $3.81(\mathrm{~s}, 1 \mathrm{H})$. The ee was determined by DAICEL Chiralpak AS (4.6 $\mathrm{mm} \phi, 250 \mathrm{~mm}$ ) [n-hexane/isopropanol $(80 / 1)(\mathrm{v} / \mathrm{v}), 1.0 \mathrm{~mL} / \mathrm{min}$, detection at $210 \mathrm{~nm}, 6.5 \mathrm{~min}$ for $(S)-13$ and $7.5 \mathrm{~min}$ for (R)-13].

\subsubsection{2 (1R,2R)-1-Isopropylcyclohexane-cis-1,2-diol (7)}

White solid; $\mathrm{mp} 91-92^{\circ} \mathrm{C}$; IR (neat) v 3400, 2940, 1449, $1071 \mathrm{~cm}^{-1} ;{ }^{1} \mathrm{H}$ NMR $(300 \mathrm{MHz}$, $\left.\mathrm{CDCl}_{3}\right) \delta 0.91(\mathrm{~d}, J=7.5 \mathrm{~Hz}, 3 \mathrm{H}), 0.94(\mathrm{~d}, J=7.5 \mathrm{~Hz}, 3 \mathrm{H}), 1.12-1.30(\mathrm{~m}, 3 \mathrm{H}), 1.40-1.80$ 
$(\mathrm{m}, 7 \mathrm{H}), 2.08(\mathrm{sep}, J=7.1 \mathrm{~Hz}, 1 \mathrm{H}), 3.66(\mathrm{dd}, J=4.2 \mathrm{~Hz}, 9.0 \mathrm{~Hz}, 1 \mathrm{H}) ;{ }^{13} \mathrm{C}$ NMR $(100 \mathrm{MHz}$, $\left.\mathrm{CDCl}_{3}\right) \delta 16.2,17.6,20.6,23.6,28.0,30.6,32.8,71.0,75.4$; HR-EI $\left(\mathrm{M}^{+}\right)$calcd for $\mathrm{C}_{9} \mathrm{H}_{18} \mathrm{O}_{2}$ 158.1307 found 158.1267. The ee of the 1-isopropylcyclohexane-cis-1,2-diol (7) was determined by HPLC of the corresponding 2-phenylcarbamoylated compound 7’ by usual method.

\subsubsection{3 (1R,2R)-1- Hydroxy-1-isopropyl-2-phenylcarbamoyloxycyclohexane (7’)}

Light-yellow oil; $[\alpha]_{\mathrm{D}}{ }^{27}+1.8\left(c=1.0, \mathrm{CHCl}_{3}, 25 \%\right.$ ee); IR (neat) v 3300, 2942, 2867, 1707, 1601, 1545, 1231, $1061 \mathrm{~cm}^{-1} ;{ }^{1} \mathrm{H}$ NMR $\left(300 \mathrm{MHz}, \mathrm{CDCl}_{3}\right) \delta 0.92(\mathrm{~d}, J=7.2 \mathrm{~Hz}$, $3 \mathrm{H}), 0.96(\mathrm{~d}, J=7.2 \mathrm{~Hz}, 3 \mathrm{H}), 1.22-1.41(\mathrm{~m}, 2 \mathrm{H}), 1.41-2.00(\mathrm{~m}, 8 \mathrm{H}), 4.91(\mathrm{dd}, J=5.1 \mathrm{~Hz}$ $10.2 \mathrm{~Hz}, 1 \mathrm{H}), 6.75(\mathrm{~s}, 1 \mathrm{H}), 7.07(\mathrm{t}, J=6.9 \mathrm{~Hz}, 1 \mathrm{H}), 7.22-7.45(\mathrm{~m}, 4 \mathrm{H}) ;{ }^{13} \mathrm{C}$ NMR $\left(100 \mathrm{MHz}, \mathrm{CDCl}_{3}\right) \delta 16.1,17.5,20.3,23.7,27.5,28.5,33.7,75.2,25.3,118.5,123.4$, 129.0, 137.9, 152.8; HR-EI $\left(\mathrm{M}^{+}\right)$calcd for $\mathrm{C}_{16} \mathrm{H}_{23} \mathrm{NO}_{3} 277.1678$ found 277.1677. The ee was determined by DAICEL Chiralpak AS (4.6 $\mathrm{mm} \phi, 250 \mathrm{~mm}$ ) [ $n$-hexane/isopropanol $(50 / 1)(\mathrm{v} / \mathrm{v}), 1.0 \mathrm{~mL} / \mathrm{min}$, detection at $254 \mathrm{~nm}, 16 \mathrm{~min}$ for (1S, 2S)-7' and $19 \mathrm{~min}$ for $\left.(1 R, 2 R)-7^{\prime}\right]$

\subsubsection{1 (S)-2-Hydroxy-2-cyclohexylcyclohexanone (14))}

Light-yellow oil (lit. ${ }^{21}$ for racemate, $\left.\mathrm{mp} 46-46.5^{\circ} \mathrm{C}\right) ;[\alpha]_{\mathrm{D}}{ }^{29}+91.9\left(c=1.2, \mathrm{CHCl}_{3}, 81 \%\right.$ ee); ${ }^{1} \mathrm{H}$ NMR $\left(300 \mathrm{MHz}, \mathrm{CDCl}_{3}\right) \delta 1.00-1.48(\mathrm{~m}, 7 \mathrm{H}), 1.55-2.06(\mathrm{~m}, 8 \mathrm{H}), 2.06-2.18(\mathrm{~m}$, 1H), 2.36-2.57 (m, 3H), $3.85(\mathrm{~s}, 1 \mathrm{H})$. The ee was determind by DAICEL Chiralpak AD $(4.6 \mathrm{~mm} \phi, 250 \mathrm{~mm})[n$-hexane/isopropanol $(40 / 1)(\mathrm{v} / \mathrm{v}), 1.0 \mathrm{~mL} / \mathrm{min}$, detection at 210 $\mathrm{nm}, 8 \mathrm{~min}$ for $(S)-14$ and $11 \min$ for $(R)-14]$. 


\subsubsection{2 (1R,2R)-1-Cyclohexylcyclohexane-cis-1,2-diol (8)}

White solid; mp $109-110^{\circ} \mathrm{C}$; IR (neat) $v 3400,2928,1445,1075 \mathrm{~cm}^{-1} ;{ }^{1} \mathrm{H}$ NMR $\left(300 \mathrm{MHz}, \mathrm{CDCl}_{3}\right) \delta 0.95-1.90(\mathrm{~m}, 21 \mathrm{H}), 3.60-3.70(\mathrm{~m}, 1 \mathrm{H}) ;{ }^{13} \mathrm{C} \mathrm{NMR}(100 \mathrm{MHz}$, $\left.\mathrm{CDCl}_{3}\right) \delta 20.8,23.7,26.7,26.9,27.8,29.5,30.6,43.6,70.7,75.2$; HR-EI $\left(\mathrm{M}^{+}\right)$calcd for $\mathrm{C}_{12} \mathrm{H}_{22} \mathrm{O}_{3}$ 198.16208 found 198.1582. The ee of the 1-cyclohexylcyclohexane-cis-1,2-diol (8) was determined by HPLC of the corresponding 2-phenylcarbamoylated compound 8'.

\subsubsection{3 (1R,2R)-1-Hydroxy-1-cyclohexyl-2-phenylcarbamoyloxycyclohexane (8')}

White solid; $\mathrm{mp} 125^{\circ} \mathrm{C} ;[\alpha]_{\mathrm{D}}^{29}+3.5\left(c=2.5, \mathrm{CHCl}_{3}, 18 \%\right.$ ee); IR (neat) $\vee 3308,2938$, $2855,1736,1608,1549,1447,1325,1237 \mathrm{~cm}^{-1} ;{ }^{1} \mathrm{H}$ NMR $\left(300 \mathrm{MHz}, \mathrm{CDCl}_{3}\right) \delta$ $0.90-1.90(\mathrm{~m}, 20 \mathrm{H}), 4.89(\mathrm{dd}, J=4.8 \mathrm{~Hz}, 10.2 \mathrm{~Hz}, 1 \mathrm{H}), 6.76(\mathrm{~s}, 1 \mathrm{H}), 7.07(\mathrm{t}, J=7.2 \mathrm{~Hz}, 1 \mathrm{H})$ 7.28-7.43 (m, 4H); ${ }^{13} \mathrm{C}$ NMR $\left(100 \mathrm{MHz}, \mathrm{CDCl}_{3}\right) \delta 20.5,23.7,26.1,26.6,26.8,26.9$, 27.4, 27.7, 30.2, 44.6, 75.0, 75.1, 118.5, 123.4, 129.1, 137.9, 152.7; HR-EI $\left(\mathrm{M}^{+}\right)$calcd for $\mathrm{C}_{19} \mathrm{H}_{27} \mathrm{NO}_{3} 317.1991$ found 317.2000 . The ee was determined by DAICEL Chiralpak AD (4.6 mm $\phi, 250 \mathrm{~mm})[n$-hexane/isopropanol $(10 / 1)(\mathrm{v} / \mathrm{v}), 1.0 \mathrm{~mL} / \mathrm{min}$, detection at $210 \mathrm{~nm}, 9 \mathrm{~min}$ for $(1 R, 2 R)-\mathbf{8}^{\prime}$ and $12 \mathrm{~min}$ for $\left.(1 S, 2 S)-\mathbf{8}^{\prime}\right]$.

\subsubsection{N-(p-Tolylsulfonyl)piperidine-(3S,4R)-diol (15)}

White solid; $\mathrm{mp} 112^{\circ} \mathrm{C}\left(\right.$ lit. $^{22}$ for racemate, $\left.\mathrm{mp} 138-140^{\circ} \mathrm{C}\right) ;[\alpha]_{\mathrm{D}}{ }^{22}-7.1\left(c=1.0, \mathrm{CHCl}_{3}\right.$, $41 \%$ ee); IR (neat) v 3350, 2926, 2872, 2359, 1929, 1732, 1661, $1599 \mathrm{~cm}^{-1} ;{ }^{1} \mathrm{H}$ NMR $\left(300 \mathrm{MHz}, \mathrm{CDCl}_{3}\right) \delta 1.77-1.95(\mathrm{~m}, 2 \mathrm{H}), 2.08-2.18(\mathrm{~m}, 1 \mathrm{H}), 2.18-2.36(\mathrm{~m}, 1 \mathrm{H}), 2.44(\mathrm{~s}$, 3H), 2.94-3.10 (m, 3H), 3.10-3.23 (m, 1H), 3.70-3.82 (m, 1H), 3.82-3.94 (m, 1H), 7.33 $(\mathrm{d}, J=8.1 \mathrm{~Hz}, 2 \mathrm{H}), 7.65(\mathrm{~d}, J=8.1 \mathrm{~Hz}, 2 \mathrm{H}) ;{ }^{13} \mathrm{C} \mathrm{NMR}\left(100 \mathrm{MHz}, \mathrm{CDCl}_{3}\right) \delta 21.5,29.2,41.9$, 
42.0, 67.4, 67.7, 127.6, 129.8, 143.8; HR-EI $\left(\mathrm{M}^{+}\right)$calcd for $\mathrm{C}_{12} \mathrm{H}_{17} \mathrm{NO}_{4} \mathrm{~S} 271.0878$ found 271.0876. The ee was determined by DAICEL Chiralcel OD-H (4.6 mm $\phi, 250$ $\mathrm{mm})[n$-hexane/ethanol $(20 / 1)(\mathrm{v} / \mathrm{v}), 1.0 \mathrm{~mL} / \mathrm{min}$, detection at $210 \mathrm{~nm}, 12 \mathrm{~min}$ for (3R,4S)- 15 and $16 \min$ for $(3 S, 4 R)-15]$.

\subsubsection{2 (3R)-Hydroxy- $N$-(p-tolylsulfonyl)piperidine-4-one (17)}

Colorless solid; $\mathrm{mp} 114^{\circ} \mathrm{C} ;[\alpha]_{\mathrm{D}}{ }^{19}+1.1\left(\mathrm{c}=0.75, \mathrm{CHCl}_{3}, 49 \%\right.$ ee); IR (neat) $v 3350$, 2926, 2857, 1732, $1345 \mathrm{~cm}^{-1} ;{ }^{1} \mathrm{H}$ NMR $\left(300 \mathrm{MHz}, \mathrm{CDCl}_{3}\right) \delta 2.30-2.43(\mathrm{~m}, 1 \mathrm{H}), 2.44$ (s, 3H), 2.50-2.90 (m, 3H), 3.53 (br s, 1H), 4.10-4.25 (m, 1H), 4.28-4.45 (m, 2H), 7.35 (d, $J=10.4 \mathrm{~Hz}, 2 \mathrm{H}), 7.69(\mathrm{~d}, J=10.4 \mathrm{~Hz}, 2 \mathrm{H}) ;{ }^{13} \mathrm{C} \mathrm{NMR}\left(100 \mathrm{MHz}, \mathrm{CDCl}_{3}\right) \delta 21.5,38.7,46.5$, $52.5,72.5,100.6,127.4,127.7,130.0,133.5,144.3$; HR-EI $\left(\mathrm{M}^{+}\right)$calcd for $\mathrm{C}_{12} \mathrm{H}_{15} \mathrm{NO}_{4} \mathrm{~S}$ 269.0722 found 269.0719. The ee was determined by DAICEL Chiralcel OJ (4.6 mm $\phi$, $250 \mathrm{~mm}) \quad$ [n-hexane/isopropanol (20/1) $(\mathrm{v} / \mathrm{v}), 1.0 \mathrm{~mL} / \mathrm{min}$, detection at 210nm, $9 \mathrm{~min}$ for (3S)-17 and $11 \min$ for (3R)-17]

\subsubsection{N-(t-Butoxycarbonyl)-3-phenylpiperidine-(3S,4S)-diol (18)}

Colorless solid; $\mathrm{mp} 101-103^{\circ} \mathrm{C} ;[\alpha]_{\mathrm{D}}^{22}-10.1\left(c=0.2, \mathrm{CHCl}_{3}, 55 \%\right.$ ee); IR (neat) v 3350, 2976, 2926, 2957, 2855, 1750, 1694, 1671, 1466, 1377, $1167 \mathrm{~cm}^{-1} ;{ }^{1} \mathrm{H}$ NMR (400MHz, $\left.\mathrm{CDCl}_{3}\right) \delta 1.48(\mathrm{~s}, 9 \mathrm{H}), 1.85-2.10(\mathrm{~m}, 3 \mathrm{H}), 2.70-2.87(\mathrm{~m}, 1 \mathrm{H}), 2.90-3.08(\mathrm{~m}, 2 \mathrm{H})$ $3.80-4.30(\mathrm{~m}, 3 \mathrm{H}), 7.28(\mathrm{t}, J=7.6 \mathrm{~Hz}, 1 \mathrm{H}), 7.38(\mathrm{t}, J=7.6 \mathrm{~Hz}, 2 \mathrm{H}), 7.47(\mathrm{~d}, J=7.6 \mathrm{~Hz}, 2 \mathrm{H})$; ${ }^{13} \mathrm{C}$ NMR $\left(100 \mathrm{MHz}, \mathrm{CDCl}_{3}\right) \delta 28.4,37.9,40.0,44.4,70.9,74.3,79.9,125.0,127.4$, 128.6, 144.8, 154.8; HR-EI $\left(\mathrm{M}^{+}\right)$calcd for $\mathrm{C}_{16} \mathrm{H}_{23} \mathrm{NO}_{4} 293.1627$ found 293.1616. The ee was determined by DAICEL Chiralcel OJ $(4.6 \mathrm{~mm} \phi, 250 \mathrm{~mm}) \quad[n$-hexane/ethanol $(15 / 1)(\mathrm{v} / \mathrm{v}), 1.0 \mathrm{~mL} / \mathrm{min}$, detection at $210 \mathrm{~nm}, 9 \mathrm{~min}$ for $(3 S, 4 S)-18$ and $11 \mathrm{~min}$ for 
$(3 R, 4 R)-18]$.

4.4.9.2 N-(t-Butoxycarbonyl)-N-(3-oxo-3-phenylpropyl)formamide (20)

Colorless oil; IR (neat) v 2978, 2932, 2359, 2344, 1740, 1686, 1342, $1149 \mathrm{~cm}^{-1} ;{ }^{1} \mathrm{H}$ NMR $\left(300 \mathrm{MHz}, \mathrm{CDCl}_{3}\right) \delta 1.53(\mathrm{~s}, 9 \mathrm{H}), 3.25(\mathrm{t}, J=7.5 \mathrm{~Hz}, 2 \mathrm{H}), 4.05(\mathrm{t}, J=7.5 \mathrm{~Hz}, 2 \mathrm{H})$, 7.40-7.50 (m, 2H), 7.50-7.64 (m, 1H), 7.90-8.00 (m, 2H), $9.19(\mathrm{~s}, 1 \mathrm{H}) ;{ }^{13} \mathrm{C} \mathrm{NMR}$ $\left(100 \mathrm{MHz}, \mathrm{CDCl}_{3}\right) \delta 28.0,29.7,36.6,36.9,84.4,128.1,128.7,133.3,136.5,163.0$ 197.7; HR-EI $\left(\mathrm{M}^{+}\right)$calcd for $\mathrm{C}_{15} \mathrm{H}_{19} \mathrm{NO}_{4} 277.1314$ found 277.1292 .

4.4.9.3 N-(t-Butoxycarbonyl)-N-(3-oxo-3-phenylpropyl)glycine methyl ester (21)

Colorless oil; IR (neat) $v$ 2976, 2359, 1752, 1705, 1682, 1367, 1213, $1169 \mathrm{~cm}^{-1} ;{ }^{1} \mathrm{H}$ NMR $\left(400 \mathrm{MHz}, \mathrm{CDCl}_{3}\right) \delta 1.41$ and $1.47(2 \mathrm{~s}, 9 \mathrm{H}), 3.25-3.45(\mathrm{~m}, 2 \mathrm{H}), 3.60-3.80(\mathrm{~m}, 5 \mathrm{H})$, $4.07(\mathrm{~d}, J=14.4 \mathrm{~Hz}, 2 \mathrm{H}), 7.40-7.55(\mathrm{~m}, 2 \mathrm{H}), 7.50-7.65(\mathrm{~m}, 1 \mathrm{H}), 7.97$ (d, J=8.0Hz, 2H); ${ }^{13} \mathrm{C}$ NMR $\left(100 \mathrm{MHz}, \mathrm{CDCl}_{3}\right) \delta 28.2,38.0,44.4,51.2,51.9,80.4,128.1,128.6,133.2$, 136.7, 155.2, 170.9, 199.2; HR-EI $\left(\mathrm{M}^{+}\right)$calcd for $\mathrm{C}_{17} \mathrm{H}_{23} \mathrm{NO}_{5} 321.1576$ found 321.1571 .

\subsubsection{1 (R)-3-Ethyl-3-hydroxy- $N$-(p-tolylsulfonyl)piperidine-4-one (23)}

White solid; $\mathrm{mp} 85-87^{\circ} \mathrm{C} ;[\alpha]_{\mathrm{D}}{ }^{27}-11.2\left(c=1.0, \mathrm{CHCl}_{3}, 31 \%\right.$ ee); IR (neat) $v 2855,1718$, 1339, $1157 \mathrm{~cm}^{-1} ;{ }^{1} \mathrm{H}$ NMR $\left(400 \mathrm{MHz}, \mathrm{CDCl}_{3}\right) \delta 0.87(\mathrm{t}, J=7.6 \mathrm{~Hz}, 3 \mathrm{H}), 1.85-2.00(\mathrm{~m}$, $1 \mathrm{H}), 2.00-2.15(\mathrm{~m}, 1 \mathrm{H}), 2.31(\mathrm{~d}, J=11.6 \mathrm{~Hz}, 1 \mathrm{H}), 2.40-2.62(\mathrm{~m}, 2 \mathrm{H}), 2.43(\mathrm{~s}, 3 \mathrm{H})$, 2.82-2.97 (m, 1H), $3.72(\mathrm{~s}, 1 \mathrm{H},-\mathrm{OH}), 4.03(\mathrm{dd}, J=2.8 \mathrm{~Hz}, J=12.0 \mathrm{~Hz}, 1 \mathrm{H}), 4.08-4.20(\mathrm{~m}$, 1H), $7.34(\mathrm{~d}, J=8.4 \mathrm{~Hz}, 2 \mathrm{H}), 7.65(\mathrm{~d}, J=8.4 \mathrm{~Hz}, 2 \mathrm{H}) ;{ }^{13} \mathrm{C} \mathrm{NMR}\left(100 \mathrm{MHz}, \mathrm{CDCl}_{3}\right) \delta 6.6$, 21.5, 29.5, 37.3, 46.9, 55.8, 78.0, 127.4, 129.9, 133.1, 144.2, 209.9; HR-EI (M $\left.{ }^{+}\right)$calcd for $\mathrm{C}_{14} \mathrm{H}_{19} \mathrm{NO}_{4} \mathrm{~S} 297.1035$ found 297.1022. The ee was determined by DAICEL 
Chiralcel OD-H (4.6 mm $\phi, 250 \mathrm{~mm})$ [n-hexane/ethanol $(20 / 1)(\mathrm{v} / \mathrm{v}), 1.0 \mathrm{~mL} / \mathrm{min}$, detection at $210 \mathrm{~nm}, 18 \mathrm{~min}$ for (3S)-23 and $19 \mathrm{~min}$ for (3R)-23].

\subsubsection{N-(p-Tolylsulfonyl)-3-ethylpiperidine-(3S,4R)-diol (22)}

White solid; $\mathrm{mp} 98-102^{\circ} \mathrm{C} ;[\alpha]_{\mathrm{D}}^{28}+3.6\left(c=0.6, \mathrm{CHCl}_{3}, 39 \%\right.$ ee $)$; IR (neat) $v 2967,1464$, 1335, $1163 \mathrm{~cm}^{-1} ;{ }^{1} \mathrm{H}$ NMR $\left(400 \mathrm{MHz}, \mathrm{CDCl}_{3}\right) \delta 0.94(\mathrm{t}, J=7.6 \mathrm{~Hz}, 3 \mathrm{H}), 1.58-1.80(\mathrm{~m}$, 2H), 1.70-1.82 (m, 1H), 1.88-2.00 (m, 1H), 2.00-2.40 (m, 2H, -OH), $2.44(\mathrm{~s}, 3 \mathrm{H}), 2.71$ $(\mathrm{d}, J=11.6 \mathrm{~Hz}, 1 \mathrm{H}), 2.70-2.82(\mathrm{~m}, 1 \mathrm{H}), 3.11(\mathrm{~d}, J=11.6 \mathrm{~Hz}, 1 \mathrm{H}), 3.18-3.30(\mathrm{~m} 1 \mathrm{H})$, 3.35-3.45 (m, 1H), $7.34(\mathrm{~d}, J=8.0 \mathrm{~Hz}, 2 \mathrm{H}), 7.64(\mathrm{~d}, J=8.0 \mathrm{~Hz}, 2 \mathrm{H}) ;{ }^{13} \mathrm{C} \mathrm{NMR}(100 \mathrm{MHz}$, $\left.\mathrm{CDCl}_{3}\right) \delta 7.0,21.5,27.7,29.5,43.2,51.0,70.5,71.8,127.6,129.8,132.9,143.8$; HR-EI $\left(\mathrm{M}^{+}\right)$calcd for $\mathrm{C}_{14} \mathrm{H}_{21} \mathrm{NO}_{4} \mathrm{~S} 299.1191$ found 299.1181. The ee was determined by DAICEL Chiralcel OD-H (4.6 mm $\phi, 250 \mathrm{~mm}) \quad$ [n-hexane/ethanol (10/1) (v/v), $1.0 \mathrm{~mL} / \mathrm{min}$, detection at $210 \mathrm{~nm}, 19 \mathrm{~min}$ for $(3 R, 4 S)-22$ and $23 \mathrm{~min}$ for $(3 S, 4 R)-22]$.

\subsection{A typical procedure for preparation of racemic aminoalcohols}

Sodium tetrahydroborate $(120 \mathrm{mmol}, 4.54 \mathrm{~g})$ and calcium chloride $(60 \mathrm{mmol}, 6.66$ g) was added to a stirred solution of racemic $N$-benzoylalanine methyl ester (racemic-30, $30 \mathrm{mmol}, 6.22 \mathrm{~g})$ in THF-MeOH $(4: 1,150 \mathrm{~mL})$ at $0^{\circ} \mathrm{C}$. The reaction mixture was stirred for $12 \mathrm{~h}$. Water $(50 \mathrm{~mL})$ was added to the resulting mixture and the solution stirred for 15 min. The solution was evaporated in vacuo to give a residue, which was then dissolved in AcOEt. The organic portion was extracted with AcOEt and dried over $\mathrm{MgSO}_{4}$. The resulting solution was concentrated in vacuo. The residue was chromatographed on silica gel $(n$-hexane/AcOEt $=1 / 1)$ to afford 26 ( $85 \%$ yield $)$. 
4.6 A typical procedure for preparation of racemic aminoaldehydes

To a solution of $\mathrm{N}$-Benzoyl-2-(hydroxymethyl)piperidine (racemic-24, $10 \mathrm{mmol}, 2.19$ g) and TEMPO $(1 \mathrm{mmol}, 156 \mathrm{mg})$ in $\mathrm{CH}_{2} \mathrm{Cl}_{2}(10 \mathrm{~mL})$ was added $\mathrm{PhI}(\mathrm{OAc})_{2}(11 \mathrm{mmol}$, $3.54 \mathrm{~g}$ ). The reaction mixture was stirred for $1 \mathrm{~h}$, then quenched with sat. aqueous $\mathrm{Na}_{2} \mathrm{~S}_{2} \mathrm{O}_{3}(5 \mathrm{~mL})$. The organic portion was extracted with $\mathrm{CH}_{2} \mathrm{Cl}_{2}$ and dried over $\mathrm{MgSO}_{4}$. The resulting solution was concentrated in vacuo. The residue was chromatographed on silica gel $(n$-hexane/AcOEt $=2 / 1)$ to afford $32(73 \%$ yield $)$.

\subsubsection{N-Benzoyl-2-piperidinecarbaldehyde $(32)^{23}$}

Colorless oil; ${ }^{1} \mathrm{H}-\mathrm{NMR}\left(300 \mathrm{MHz}, \mathrm{CDCl}_{3}\right) \delta$ 1.30-1.90 (m, 5H), 2.16-2.20 (m, 1H), 3.02-3.21 (m, 1H), 3.60-3.80 (m, 1H), $5.30(\mathrm{~s}, 1 \mathrm{H}), 7.43(\mathrm{~m}, 5 \mathrm{H}), 9.66(\mathrm{~s}, 1 \mathrm{H})$.

\subsubsection{N-Benzoyl-2-pyrrolidinecarbaldehyde $(33)^{23}$}

Colorless oil; ${ }^{1} \mathrm{H}-\mathrm{NMR}\left(300 \mathrm{MHz}, \mathrm{CDCl}_{3}\right) \delta$ 1.81-2.30 (m, 4H), 3.50-3.70 (m, 2H), 4.64-4.75 (m, 1H), 7.30-7.45 (m, 3H), 7.50-7.80 (m, 2H), $9.69(\mathrm{~s}, 1 \mathrm{H})$.

\subsubsection{N-(1-Formylethyl)benzamide (34) $)^{24}$}

Colorless oil; ${ }^{1} \mathrm{H}-\mathrm{NMR}\left(300 \mathrm{MHz}, \mathrm{CDCl}_{3}\right) \delta, 1.51$ (d, J=7.5Hz, 3H), 4.70-4.81 (m, 1H), 6.83 (br s, 1H), 7.32-7.58 (m, 3H), 7.81-7.86 (m, 2H), $9.66(\mathrm{~s}, 1 \mathrm{H})$.

\subsubsection{N-(1-Formyl-2-methylpropyl)benzamide (35)}

White solid; $\mathrm{mp} 68-70^{\circ} \mathrm{C}$; IR (neat) $v$ 3320, 2967, 1732, 1647, 1536, $1314 \mathrm{~cm}^{-1}$; ${ }^{1} \mathrm{H}-\mathrm{NMR}\left(300 \mathrm{MHz}, \mathrm{CDCl}_{3}\right) \delta 1.07$ (d, J=6.9Hz, 3H), 1.09 (d, J=6.9Hz, 3H), 2.40-2.52 (m, 1H), 4.82-4.89 (m, 1H), 6.73 (br s, 1H), 7.44-7.59 (m, 3H), 7.83 (d, J=6.9Hz, 2H), 
$9.77(\mathrm{~s}, 1 \mathrm{H}) ;{ }^{13} \mathrm{C} \mathrm{NMR}\left(100 \mathrm{MHz}, \mathrm{CDCl}_{3}\right) \delta 17.9,19.0,24.4,63.6,127.1,128.6,131.8$, 140.0, 167.6, 199.8; HR-EI $\left(\mathrm{M}^{+}\right)$calcd for $\mathrm{C}_{12} \mathrm{H}_{15} \mathrm{NO}_{2} 205.1103$ found 205.1098.

\subsection{Asymmetric electrochemical oxidation of aminoalcohols}

The oxidation were carried out according to a typical experimental procedure for the oxidation of 1.

4.7.1.1 N-Benzoyl-(2R)-(methoxycarbonyl)piperidine (28) (lit. ${ }^{23}$ for racemate)

Colorless oil; $[\alpha]_{\mathrm{D}}{ }^{26}+51.0\left(c=0.40, \mathrm{CHCl}_{3}, 87 \%\right.$ ee $) ;{ }^{1} \mathrm{H}-\mathrm{NMR}\left(300 \mathrm{MHz}, \mathrm{CDCl}_{3}\right) \delta$ 1.25-1.82 (m, 5H) 2.26-2.40 (m, 1H), 3.18-3.30 (m, 1H), 3.60-3.70 (m, 1H), $3.79(\mathrm{~s}$, 3H), 5.50-5.58 $(\mathrm{m}, 1 \mathrm{H}), 7.42(\mathrm{~m}, 5 \mathrm{H})$. The ee was obtained by DAICEL Chiralcel OD-H (4.6 mm $\phi, 250 \mathrm{~mm}) \quad$ [n-hexane/isopropanol (30/1) (v/v), $1.0 \mathrm{~mL} / \mathrm{min}$, detection at $254 \mathrm{~nm}, 25 \mathrm{~min}$ for $(R)-\mathbf{2 8}$ and $27 \mathrm{~min}$ for $(S)-\mathbf{2 8}]$.

\subsubsection{N-Benzoyl-(2S)-(hydroxymethyl)piperidine $(24)^{4 \mathrm{c}}$}

White solid; mp $89-92^{\circ} \mathrm{C}\left(1 \mathrm{it} .{ }^{25}\right.$ for racemate, mp $\left.93-95^{\circ} \mathrm{C}\right) ;[\alpha]_{\mathrm{D}}{ }^{26}-7.1\left(c=2.0, \mathrm{CHCl}_{3}\right.$, $12 \%$ ee); ${ }^{1} \mathrm{H}-\mathrm{NMR}\left(300 \mathrm{MHz}, \mathrm{CDCl}_{3}\right) \delta 1.40-1.90(\mathrm{~m}, 6 \mathrm{H})$ 2.80-3.20 (m, 2H), 3.50-3.80 $(\mathrm{m}, 1 \mathrm{H}), 3.96(\mathrm{dd}, J=9.9,10.5 \mathrm{~Hz}, 1 \mathrm{H}), 4.85($ br s, $1 \mathrm{H}), 7.41(\mathrm{~m}, 5 \mathrm{H})$. The ee was determined by DAICEL Chiralcel OD-H (4.6 mm $\phi, 250 \mathrm{~mm}) \quad$ [n-hexane/isopropanol $(30 / 1)(\mathrm{v} / \mathrm{v}), 1.0 \mathrm{~mL} / \mathrm{min}$, detection at $254 \mathrm{~nm}, 30 \mathrm{~min}$ for $(S)-24$ and $34 \mathrm{~min}$ for $(R)-24]$.

4.7.2.1 N-Benzoyl-(2R)-methoxycarbonylpyrrolidine (N-Benzoyl-D-proline methyl ester) (29)

White solid; mp $69-72^{\circ} \mathrm{C}$ (lit. ${ }^{26}$ for L-form, mp $\left.89-90^{\circ} \mathrm{C}\right) ;[\alpha]_{\mathrm{D}}{ }^{21}+83.7\left(c=0.5, \mathrm{CHCl}_{3}\right.$, 
$86 \%$ ee); ${ }^{1} \mathrm{H}-\mathrm{NMR}\left(300 \mathrm{MHz}, \mathrm{CDCl}_{3}\right) \delta 1.80-2.11(\mathrm{~m}, 3 \mathrm{H}), 2.14-2.40(\mathrm{~m}, 1 \mathrm{H}), 3.50-3.60$ (m, 1H), 3.62-3.71 (m, 1H), $3.78(\mathrm{~s}, 3 \mathrm{H}), 4.66-4.70(\mathrm{~m}, 1 \mathrm{H}), 7.30-7.45(3 \mathrm{H}), 7.50-7.60$ $(\mathrm{m}, 2 \mathrm{H})$. The ee was determined by DAICEL Chiralpak AD (4.6 mm $\phi, 250 \mathrm{~mm})$ [n-hexane/isopropanol (20/1) (v/v), $1.0 \mathrm{~mL} / \mathrm{min}$, detection at $254 \mathrm{~nm}, 30 \mathrm{~min}$ for $(R)-29$ and $39 \min$ for $(S)-29]$.

\subsubsection{N-Benzoyl-(2S)-hydroxymethylpyrrolidine $(25)^{27}$}

Colorless oil; $[\alpha]_{\mathrm{D}}{ }^{19}-21.1\left(c=1.6, \mathrm{CHCl}_{3}, 15 \%\right.$ ee); ${ }^{1} \mathrm{H}-\mathrm{NMR}\left(300 \mathrm{MHz}, \mathrm{CDCl}_{3}\right) \delta$ 1.57-1.95 (m, 4H) 2.12-2.25 (m, 1H), 3.42-3.58 (m, 2H), 3.70-3.83 (m, 2H), 4,37-4.46 $(\mathrm{m}, 1 \mathrm{H}), 4.90-4.98(\mathrm{~m}, 1 \mathrm{H}), 7.38-7.55(\mathrm{~m}, 5 \mathrm{H})$. The ee was obtained by DAICEL Chiralpak AD (4.6 mm $\phi, 250 \mathrm{~mm}) \quad$ [n-hexane/isopropanol (20/1) (v/v), $1.0 \mathrm{~mL} / \mathrm{min}$, detection at $254 \mathrm{~nm}, 30 \mathrm{~min}$ for $(R)-25$ and $36 \mathrm{~min}$ for $(S)-25]$.

4.7.3.1 Methyl (2R)-(N-benzoyl)aminopropionate ( $N$-Benzoyl-D-alanine methyl ester) (30)

Colorless oil (lit. ${ }^{28}$ for L-form, mp $\left.52-53^{\circ} \mathrm{C}\right) ;[\alpha]_{\mathrm{D}}{ }^{23}-23.3\left(c=1.4, \mathrm{CHCl}_{3}, 59 \%\right.$ ee) $\left[\right.$ lit. $^{28}$ for L-form, $\left.[\alpha]_{\mathrm{D}}^{23}+38.6\left(c=2.9, \mathrm{CHCl}_{3}\right)\right]$; ${ }^{1} \mathrm{H}-\mathrm{NMR}\left(300 \mathrm{MHz}, \mathrm{CDCl}_{3}\right) \delta 1.53(\mathrm{~d}$, $J=6.9 \mathrm{~Hz}, 3 \mathrm{H}), 3.80(\mathrm{~s}, 3 \mathrm{H}), 4.75-4.83(\mathrm{~m}, 1 \mathrm{H}), 6.74($ br s, $1 \mathrm{H}), 7.41-7.55(\mathrm{~m}, 3 \mathrm{H})$, 7.80-7.85 (m, 2H). The ee was obtained by DAICEL Chiralcel OJ-H (4.6 mm $\phi, 250$ $\mathrm{mm})[n$-hexane/isopropanol $(20 / 1)(\mathrm{v} / \mathrm{v}), 1.0 \mathrm{~mL} / \mathrm{min}$, detection at $220 \mathrm{~nm}, 16 \mathrm{~min}$ for (R)-30 and $17 \mathrm{~min}$ for $(S)-30]$.

\subsubsection{2 (2S)-(N-Benzoyl)aminopropan-1-ol (26) $)^{29}$}

White solid; mp $110-112^{\circ} \mathrm{C}\left(\right.$ lit. $\left.^{29} \mathrm{mp} 132-133^{\circ} \mathrm{C}\right) ; \quad[\alpha]_{\mathrm{D}}{ }^{26}-1.0\left(c=0.9, \mathrm{CHCl}_{3}, 18 \%\right.$ ee $)$; 
${ }^{1} \mathrm{H}-\mathrm{NMR}\left(300 \mathrm{MHz}, \mathrm{CDCl}_{3}\right) \delta 1.28(\mathrm{~d}, \mathrm{~J}=6.6 \mathrm{~Hz}, 3 \mathrm{H}), 2.40-2.83$ (br s, $\left.1 \mathrm{H}\right), 3.60-3.68(\mathrm{~m}$, 1H), 3.72-3.80 (m, 1H), 4.322-4.35 (m, 1H), 6.44 (br s, 1H), 7.30-7.56 (m, 3H), 7.70-7.88 (m, 2H). The ee was determined by DAICEL Chiralcel OJ-H (4.6 mm $\phi, 250$ $\mathrm{mm})[\mathrm{n}$-hexane/isopropanol $(20 / 1)(\mathrm{v} / \mathrm{v}), 1.0 \mathrm{~mL} / \mathrm{min}$, detection at $220 \mathrm{~nm}, 8.3 \mathrm{~min}$ for (S)-26 and 9.6 min for $(R)-\mathbf{2 6}]$.

4.7.4.1 Methyl (2R)-(N-benzoyl)amino-3-methylbutyrate ( $N$-Benzoyl-D-valine methyl ester) (31)

White solid; mp $94-96^{\circ} \mathrm{C}$ (lit. ${ }^{30}$ for racemic form, mp $\left.86^{\circ} \mathrm{C}\right)$; $[\alpha]_{\mathrm{D}}{ }^{20}-33.5\left(c=0.5, \mathrm{CHCl}_{3}\right.$, $64 \%$ ee); ${ }^{1} \mathrm{H}-\mathrm{NMR}\left(300 \mathrm{MHz}, \mathrm{CDCl}_{3}\right) \delta 0.99(\mathrm{~d}, J=7.2 \mathrm{~Hz}, 3 \mathrm{H}), 1.03(\mathrm{~d}, J=7.2 \mathrm{~Hz}, 3 \mathrm{H})$, 2.22-2.35 (m, 1H), $3.78(\mathrm{~s}, 3 \mathrm{H}), 4.78-4.82(\mathrm{~m}, 1 \mathrm{H}), 6.62(\mathrm{~m}, 1 \mathrm{H}), 7.43-7.56(\mathrm{~m}, 3 \mathrm{H})$, $7.82(\mathrm{~d}, J=6.6 \mathrm{~Hz}, 2 \mathrm{H})$. The ee was obtained by DAICEL Chiralcel OD-H (4.6 $\mathrm{mm} \phi$, $250 \mathrm{~mm})[n$-hexane/isopropanol $(50 / 1)(\mathrm{v} / \mathrm{v}), 1.0 \mathrm{~mL} / \mathrm{min}$, detection at $254 \mathrm{~nm}, 15$ $\min$ for $(R)-31$ and $20 \min$ for $(S)-31]$.

4.7.4.2 (2S)-(N-Benzoyl)amino-3-methylbutan-1-ol (27) ${ }^{31}$

White solid; mp $84-85^{\circ} \mathrm{C}\left(\right.$ lit. $\left.{ }^{31} \mathrm{mp} 99^{\circ} \mathrm{C}\right)$; $[\alpha]_{\mathrm{D}}{ }^{27}-4.5\left(c=1.0, \mathrm{CHCl}_{3}, 12 \%\right.$ ee); ${ }^{1} \mathrm{H}-\mathrm{NMR}$ $\left(300 \mathrm{MHz}, \mathrm{CDCl}_{3}\right) \delta 1.00(\mathrm{~d}, J=6.9 \mathrm{~Hz}, 3 \mathrm{H}) 1.02(\mathrm{~d}, J=6.9 \mathrm{~Hz}, 3 \mathrm{H}), 1.95-2.06(\mathrm{~m}, 1 \mathrm{H})$, 2.90-3.22 (br s, $1 \mathrm{H}), 3.75-3.78(\mathrm{~m}, 2 \mathrm{H}), 3.89-3.98(\mathrm{~m}, 1 \mathrm{H}), 6.48$ (br s, $1 \mathrm{H}), 7.39-7.56$ $(\mathrm{m}, 3 \mathrm{H}), 7.77(\mathrm{~d}, J=6.9 \mathrm{~Hz}, 2 \mathrm{H})$. The ee was obtained by DAICEL Chiralcel OD-H (4.6 $\mathrm{mm} \phi, 250 \mathrm{~mm}) \quad[n$-hexane/isopropanol $(20 / 1)(\mathrm{v} / \mathrm{v}), 1.0 \mathrm{~mL} / \mathrm{min}$, detection at $254 \mathrm{~nm}$, $13 \min$ for $(S)-27$ and $22 \min$ for $(R)-27]$.

\section{Acknowledgements}


We thank JSPS Research Fellowships for Young Scientists, the Sumitomo Foundation, and a Grant-in-Aid for Young Scientists (B) (19790017) from the Ministry of Education, Science, Sports and Culture, Japan.

\section{References and notes}

1. (a) David, S. C. R. Acad. Sci. Paris, Serie C 1974, 278, 1051-1053. (b) David, S.; Theffry, A. J. Chem. Soc. Perkin 1 1979, 1568-1573.

2. Maki, T.; Fukae, K.; Harasawa, H.; Ohishi, T.; Matsumura, Y.; Onomura, O. Tetrahedron Lett. 1998, 39, 651-654.

3. Onomura, O.; Arimoto, H.; Matsumura, Y.; Demizu, Y. Tetrahedron Lett. 2007, 48, 8668-8672.

4. Some of asymmetric reactions catalyzed with $\mathrm{Cu}(\mathrm{II})-\mathrm{Ph}-\mathrm{BOX}$ reported by us: Monobenzoylation of 1,2-diols; (a) Matsumura, Y.; Maki, T.; Murakami, S.; Onomura. O. J. Am. Chem. Soc. 2003, 125, 2052-2053. Monosulfonylation of 1,2-diols; (b) Demizu, Y.; Matsumoto, K.; Onomura, O.; Matsumura, Y. Tetrahedron Lett. 2007, 48, 7605-7609. Benzoylation of 1,2-aminoalcohols; (c) Mitsuda, M.; Tanaka, T.; Tanaka, T.; Demizu, Y.; Onomura, O.; Matsumura, Y. Tetrahedron Lett. 2006, 47, 8073-8077. Tosylation of 2-hydroxyalkanamides; (d) Onomura, O.; Mitsuda, M.; Nguyen, M. T. T.; Demizu, Y. Tetrahedron Lett. 2007, 48, 9080-9084. Benzoylation of 3-hydroxyalkanamides; (e) Demizu, Y.; Kubo, Y.; Matsumura, Y.; Onomura, O. Synlett. 2008, 433-437. Alkylation into iminium ions; (f) Matsumura, Y.; Minato, D.; Onomura, O. J. Organomet. Chem. 2007, 692, 654-663.

5. Jakka, K.; Zhao, C-. G. Org. Lett. 2006, 8, 3013-3015.

6. Ladziata, U.; Carlson, J.; Zhdankin, V. V. Tetrahedron Lett. 2006, 47, 6301-6304.

7. Kagan, H. B.; Fiaud, J. C. Topics in Stereochemistry; Eliel, E. L., Ed.; Wiley \& Sons: New York, 1988, Vol. 18, 249-330.

8. The absolute stereoconfiguration of recovered $(R, R)-\mathbf{1}$ was determined by comparing with specific rotation of authentic sample. ${ }^{9}$

9. Huang, J.; Corey, E. J. Org. Lett. 2003, 5, 3455-3458.

10. Absolute stereoconfigurations of $(R, R)-3-8$ shown in Eq. 5 and Table 2 were deduced on the basis of that of $(R, R)$-1ap.

11. Absolute stereoconfigurations of 15, 17, 18, 22, and 23 shown in Eqs. 6-8 were deduced on the basis of that of $(R, R)-1$.

12. Schirmann, P. J.; Matthews, R.S.; Dittmer, D. C. J. Org. Chem. 1983, 48, 4426-4427. 
13. Racemic $N$-benzyloxycarbonylpiperidinemethanol ${ }^{4 c}$ was hardly oxidized under the reaction conditions.

14. Excess amounts of electricity (10 20 F/mol for aminoalcohol 24, 5 10 F/mol for aminoaldehyde 32) did not improve yields of aminoester 28.

15. King, S. B.; Sharpless, K. B. Tetrahedron Lett. 1994, 35, 5611-5612.

16. Syper, L. Tetrahedron 1987, 43, 2853-2872.

17. Wistuba, E.; Ruechardt, C. Tetrahedron Lett. 1981, 22, 4069-72.

18. Kise, N.; Agui, S.; Morimoto, S.; Ueda, N. J. Org. Chem. 2005, 70, 9407-9410.

19. Bisel, P.; Lauktien, G.; Weckert, E.; Frahm, A. W. Tetrahedron: Asymmetry 1998, 9, 4027-4034.

20. Costantino, P.; Crotti, P.; Ferretti, M.; Macchia, F. J. Org. Chem. 1982, 47, 2917-2923.

21. Elphimoff-Felkin, I.; Le Ny, G.; Tchoubar, B. Bull. Soc. Chim. Fr. 1958, 522-531.

22. Beligny, S.; Eibauer, S.; Maechling, S.; Blechert, S. Angew. Chem. Int. Ed. Engl. 2006, 45, 1900-1903.

23. Amino, Y.; Nishi, S.; Izawa, K. Bull. Chem. Soc. Jpn. 1991, 64, 620-623.

24. Okutome, T.; Sakurai, Y.; Kurumi, M.; Kawamura, H.; Sato, S.; Yamaguchi, K. Chem. Pham. Bull. 1975, 23, 48-53.

25. Morcuende, A.; Ors, M.; Valverde, S.; Herradón, B. J. Org. Chem. 1996, 61, 5264-5270.

26. Shono, T.; Matsumura, Y.; Uchida, K.; Kanazawa, T. Tsuda, K. J. Org. Chem. 1984, 49, 3711-3716.

27. Takahashi, H.; Kawabata, A.; Higashiyama, K. Chem. Pharm. Bull. 1987, 45, 1604-1607.

28. Li, W.-R.; Yo, Y.-C.; Lin, Y.-S. Tetrahedron 2000, 56, 8867-8875.

29. Krueger, W. C.; Johnson, R. A.; Pschingoda, L. M. J. Am. Chem. Soc. 1971, 93, 4865-4872.

30. Croft, A. K.; Easton, C. J.; Radom, L. J. Am. Chem. Soc. 2003, 125, 4119-4124.

31. Menges, F.; Neuburger, M.; Pfaltz, A. Org. Lett. 2002, 4, 4713-4716. 